\title{
43. PALEOMAGNETISM OF CENOZOIC SEDIMENTS IN HOLES 762B AND 763A, CENTRAL EXMOUTH PLATEAU, NORTHWEST AUSTRALIA ${ }^{1}$
}

\author{
Cheng Tang ${ }^{2}$
}

\begin{abstract}
The magnetostratigraphy of Neogene sediments from Holes 762B and 763A are presented in this paper. Hole 762 B contains 17 reversals. All reversals above the base of the Gilbert are correlated with the magnetic polarity time scale (Haq et al., 1988). Hole 763A yields a record of about 20 reversals that can be correlated to the magnetic polarity time scale, documenting all reversals to the base of Chron $4 \mathrm{~A}$. Based on the correlation, the sediment accumulation vs. time for Holes $762 \mathrm{~B}$ and $763 \mathrm{~A}$ are determined. The age-depth curves obtained show a similar pattern of sedimentation rate since $6.8 \mathrm{Ma}$.

The study also indicates a correlation between the fluctuations in the magnetic parameters (natural remanent magnetization intensity and susceptibility), the lithologic changes, and changes in iron content at both holes. This correlation suggests that the natural remanent magnetization intensity and susceptibility changes observed in Holes $762 \mathrm{~B}$ and $763 \mathrm{~A}$ are controlled by changes in depositional processes probably associated with climatic variations.
\end{abstract}

\section{INTRODUCTION}

\section{Geologic Setting}

The Exmouth Plateau is a rifted and deeply subsided piece of continental crust covered by more than $8 \mathrm{~km}$ of Phanerozoic sediments. Two sites (762 and 763) were drilled on the western part of the central Exmouth Plateau (Fig. 1) during Leg 122. The major objectives of drilling at these sites included documenting the Cretaceous to Quaternary depositional sequences and studying the Cretaceous and Tertiary paleoenvironments of this sediment-starved passive continental margin. Over $1.63 \mathrm{~km}$ of sediments were drilled from Sites 762 and 763 , including more than $300 \mathrm{~m}$ of Neogene sediments. It has been proved since the early days of deep-sea coring that magnetostratigraphy can provide a basis for core correlation and for establishing a precise time calibration (Hays et al., 1969, Opdyke et al., 1974). This chapter summarizes the magnetostratigraphy of the Neogene sediment sequence and the susceptibility record from the Cenozoic sediment at both sites.

Hole 762B is located on the western part of the central Exmouth Plateau $\left(19^{\circ} 53^{\prime} \mathrm{S}, 112^{\circ} 15^{\prime} \mathrm{E}\right)$ at a water depth of 1360 $\mathrm{m}$. This hole was continuously cored by the advanced piston corer (APC) to a depth of 175.4 meters below seafloor (mbsf) with an excellent recovery rate of $99 \%$. The lithology of the $145 \mathrm{~m}$ of Neogene sediments is relatively simple, characterized by pelagic deposition of foraminiferal nannofossil ooze with a small amount of terrigenous material. Sedimentation rates are generally low and sedimentation is interrupted by several hiatuses (see "Biostratigraphy" section, "Site 762" chapter, Haq, von Rad, O'Connell, et al., 1990). Hole 763A is located $84 \mathrm{~km}$ south of Hole $762 \mathrm{~B}\left(20^{\circ} 19^{\prime} \mathrm{S}, 112^{\circ} 52^{\prime} \mathrm{E}\right)$ at a water depth of $1367.5 \mathrm{~m}$. Twenty APC cores were routinely taken from this hole to a depth of 185.4 mbsf with an excellent recovery rate. Hole $763 \mathrm{~A}$ comprises Quaternary through upper Eocene ooze and semi-indurated chalk, both with clay. The lithology of the $160 \mathrm{~m}$ of Neogene sediments is dominated

\footnotetext{
${ }^{1}$ von Rad, U., Haq, B. U., et al., 1992. Proc. ODP, Sci. Results, 122: College Station, TX (Ocean Drilling Program).

2 Earth Science Board, University of California, Santa Cruz, Santa Cruz, CA 95064, U.S.A.
}

by nannofossil oozes with abundant foraminifers and very small amounts of terrigenous quartz and clay minerals.

\section{Methods}

Aboard the drillship JOIDES Resolution we measured the natural remanent magnetization (NRM) of archive sections from each hole, excluding badly disturbed intervals, with a 2G-Enterprises $760 \mathrm{R}$ cryogenic magnetometer at 5 - or $10-\mathrm{cm}$ intervals. The sections were then demagnetized in a 9-mT alternating field (AF) and their remanence was measured again. Unfortunately, the AF demagnetization at $9 \mathrm{mT}$ was not sufficient to remove the common viscous remanent magnetization (VRM) overprint for a large number of core sections. For this reason, two discrete samples from each section were taken by carefully pushing plastic $7-\mathrm{cm}^{3}$ boxes into the split, visually undisturbed sections. About 335 discrete samples were taken from both holes for shore-based study.

All discrete samples were subjected to stepwise AF demagnetization at higher demagnetization levels above $35 \mathrm{mT}$ in the paleomagnetic laboratory at the University of California, Santa Cruz. If the remanence appeared unstable, additional measurements were made at higher demagnetization levels (up to $80 \mathrm{mT}$ in some cases) in an effort to isolate the stable remanence. The reported inclination and declination in Tables 1 and 2 are based on directions of demagnetization levels above $15 \mathrm{mT}$. Both shipboard measurements of archive halves and results of discrete samples were used to determine the magnetostratigraphy in Holes 762B and 763A.

Because the shipboard core-orienting device was not operating during the entire cruise, the absolute declination is not known. Consequently, the reversals were picked largely on the basis of the inclination data and confirmed by relative declination changes. The expected inclinations for the two sites (assuming an axial geocentric dipole) were $-36^{\circ}$ (normal) and $36^{\circ}$ (reversed). This inclination is sufficiently steep to determine the polarity of cleaned samples.

Measurement of volume susceptibility was made at 5- to $10-\mathrm{cm}$ intervals on whole $1.5-\mathrm{m}$ sections from Holes $762 \mathrm{~B}$ and 763A. A Bartington Instruments' MS-1 magnetic susceptibility meter with a $80-\mathrm{mm}$ loop sensor was employed. This instrument is capable of measuring susceptibility with a sensitivity of $1 \times$ $10^{-7}$ cgs. Susceptibility is controlled by changes in magnetic mineral assemblages and concentration. Although susceptibility 


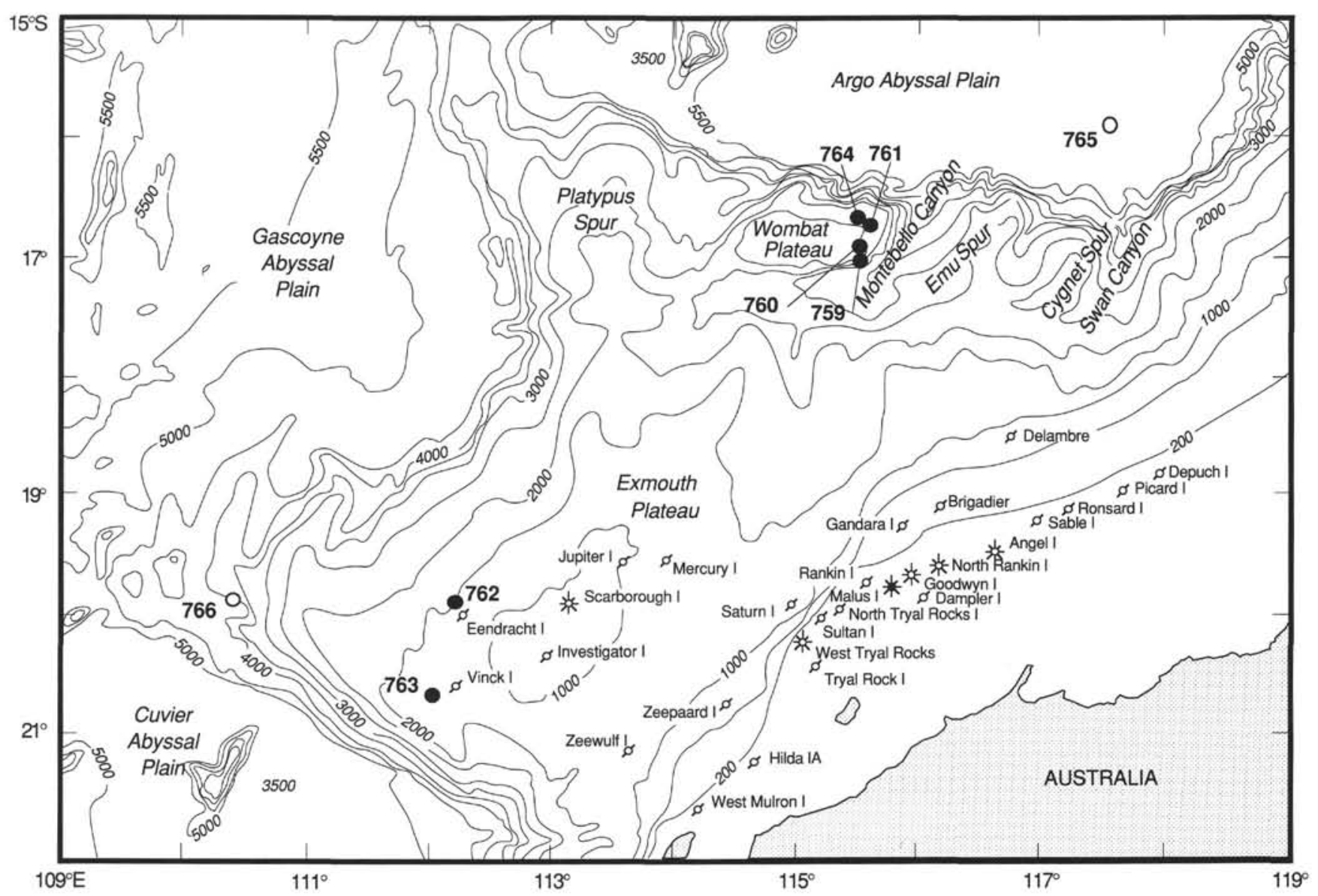

Figure 1. Bathymetric map (in meters) of the central Exmouth Plateau with location of Sites 762 and 763, as well as other Leg 122 (solid circles) and Leg 123 (open circles) drill sites. Commercial petroleum exploration wells are denoted by standard industry symbols.

is independent of magnetic mineral grain size, it is indicative of bulk magnetic mineral content when magnetic mineralogy is held constant. Whole-core susceptibility is proportional to the volume concentration of the most susceptible magnetic material that the sediment contains. Thus, the susceptibility profiles often reflect downhole variation in lithology-that is, changes in the ratio of magnetic to nonmagnetic constituents in the sediments. Therefore, whole-core susceptibility measurements have been used in previous studies to provide a rapid, nondestructive method of regional-scale lithostratigraphic correction between sites (e.g., Thompson et al., 1980; King, 1986; Robinson, 1986; Bloemendal et al., 1988; Doh et al., 1988; Hall et al., 1989; Bloemendal et al., 1989). The objective of the whole-core susceptibility measurements made at Sites 762 and 763, therefore, was to correlate Hole $762 \mathrm{~B}$ to Hole $763 \mathrm{~A}$ with a high degree of lithostratigraphic resolution.

\section{RESULTS}

\section{Demagnetization Behavior}

Figure 2 shows representative Zijderveld diagrams for samples of Neogene sediments from Holes 762B and 763A. The demagnetization curves commonly fall into one of four groups (types A-D). One group (32\% of the samples), shown in Figure 2A, has a linear demagnetization path and a median destructive field between 30 and $40 \mathrm{mT}$, which suggests a stable, single component of magnetization. In contrast, some samples (12\%) exhibit demagnetization paths similar to that in Figure 2D. This diagram indicates an unstable magnetization.
The polarity of this specimen may be reversed, though a straight line segment cannot be obtained from the plot. Most of samples $(37 \%)$ have Zijderveld diagrams similar to that in Figure 2B. These samples are characterized by the removal of a soft secondary component by $5 \mathrm{mT}$ or $9 \mathrm{mT}$, and subsequent AF demagnetization results in little or no change in the direction of magnetization but a steady decrease in intensity with increasing AF strength. Finally, some samples (19\%) behave like the one shown in Figure 2C, displaying a large normal component that is removed at $20 \mathrm{mT}$. This component is most likely a current field overprint that is viscous in origin because it is removed at low AF fields.

\section{Magnetostratigraphy}

Tables 1 and 2 list the paleomagnetic data of the discrete samples from Holes 762B and 763A. Figures 3 and 4 show the inclination and relative declination of all cleaned samples plotted against depth for Holes $762 \mathrm{~B}$ and $763 \mathrm{~A}$, respectively.

\section{Hole $762 B$}

No discrete samples were taken above 25 mbsf. The reversal boundaries within this interval were identified based on shipboard measurements of archive sections, suggesting two reversals at $11.2 \mathrm{mbsf}$ and $12.1 \mathrm{mbsf}$, respectively (see "Paleomagnetics" section, "Site 762" chapter, Haq, von Rad, O'Connell, et al., 1990). Below 25 mbsf, a combination of 100 discrete samples and the shipboard measurements yields an excellent record. This shows the polarity sequence from the base of the Gilbert at 100.1 mbsf to the upper Olduvai 
Table 1. Paleomagnetic results of discrete samples from Hole 762B.

\begin{tabular}{|c|c|c|c|c|c|c|}
\hline $\begin{array}{l}\text { Core, section, } \\
\text { interval }(\mathrm{cm})^{\mathrm{a}}\end{array}$ & $\begin{array}{l}\text { Depth } \\
\text { (mbsf) }\end{array}$ & $\begin{array}{c}\text { Declination } \\
\left({ }^{\circ}\right)\end{array}$ & $\begin{array}{c}\text { Inclination } \\
\left({ }^{\circ}\right)\end{array}$ & $\begin{array}{l}\text { NRM } \\
(\mathrm{emu})\end{array}$ & $\begin{array}{l}\text { Peak AF field } \\
(\mathrm{mT})\end{array}$ & $\begin{array}{c}\text { Demagnetization } \\
\text { type }^{b}\end{array}$ \\
\hline \multicolumn{7}{|l|}{$122-762 \mathrm{~B}-$} \\
\hline $4 \mathrm{H}-1,130$ & 24.70 & 157.10 & 49.30 & $1.13 \mathrm{E}-08$ & 80 & D \\
\hline $4 \mathrm{H}-2,130$ & 26.20 & 102.60 & -23.00 & 7.13 E-08 & 80 & A \\
\hline $4 \mathrm{H}-3,130$ & 27.70 & 359.20 & -23.60 & $2.65 \mathrm{E}-08$ & 80 & C \\
\hline $4 \mathrm{H}-4,130$ & 29.20 & 192.40 & 34.00 & $1.79 \mathrm{E}-08$ & 75 & C \\
\hline $4 \mathrm{H}-5,130$ & 30.70 & 208.30 & 40.50 & $2.01 \mathrm{E}-08$ & 75 & C \\
\hline $4 \mathrm{H}-6,130$ & 32.20 & 147.40 & 30.30 & 8.05 E-08 & 75 & B \\
\hline $5 \mathrm{H}-1,134$ & 34.24 & 145.30 & 18.20 & 4.93 E- 07 & 75 & B \\
\hline $5 \mathrm{H}-2,134$ & 35.74 & 166.00 & 53.50 & $6.18 \mathrm{E}-07$ & 75 & B \\
\hline $5 \mathrm{H}-3,134$ & 37.24 & 327.00 & 42.80 & $7.80 \mathrm{E}-07$ & 75 & B \\
\hline $5 \mathrm{H}-4,134$ & 38.74 & 187.30 & -0.20 & $1.56 \mathrm{E}-06$ & 60 & B \\
\hline $5 \mathrm{H}-5,134$ & 40.24 & 220.30 & -18.30 & $1.97 \mathrm{E}-06$ & 60 & A \\
\hline $5 \mathrm{H}-6,102$ & 41.52 & 218.90 & -62.70 & $7.11 \mathrm{E}-06$ & 60 & A \\
\hline $6 \mathrm{H}-1,130$ & 43.70 & 144.40 & -59.60 & $4.23 \mathrm{E}-06$ & 60 & $\mathrm{~A}$ \\
\hline $6 \mathrm{H}-2,130$ & 45.20 & 230.40 & -20.30 & $3.37 \mathrm{E}-06$ & 70 & B \\
\hline $6 \mathrm{H}-3,130$ & 46.70 & 197.40 & 44.40 & 6.39 E- 06 & 70 & B \\
\hline $6 \mathrm{H}-4,130$ & 48.20 & 217.00 & -28.80 & 8.91 E- 07 & 70 & $\mathrm{C}$ \\
\hline $6 \mathrm{H}-5,130$ & 49.70 & 185.30 & -49.70 & 5.25 E-06 & 70 & A \\
\hline $6 \mathrm{H}-6,130$ & 51.20 & 252.10 & -22.10 & 9.52 E-07 & 70 & B \\
\hline $6 \mathrm{H}-7,48$ & 52.70 & 45.80 & 39.40 & $4.76 \mathrm{E}-06$ & 70 & A \\
\hline $7 \mathrm{H}-1,130$ & 53.20 & 198.70 & -6.00 & 4.47 E-06 & 70 & A \\
\hline $7 \mathrm{H}-2,130$ & 54.70 & 211.30 & -22.80 & 6.01 E-06 & 70 & A \\
\hline $7 \mathrm{H}-3,130$ & 56.20 & 207.30 & -46.80 & 3.97 E-06 & 70 & B \\
\hline $7 \mathrm{H}-4,130$ & 57.70 & 176.00 & -19.30 & $1.67 \mathrm{E}-06$ & 85 & B \\
\hline $7 \mathrm{H}-5,130$ & 59.20 & 159.30 & -58.80 & 5.37 E-06 & 90 & A \\
\hline $7 \mathrm{H}-6,130$ & 60.70 & 172.70 & -73.90 & 1.01 E-05 & 90 & A \\
\hline $7 \mathrm{H}-7,42$ & 61.23 & 70.50 & -45.00 & 4.32 E- 06 & 90 & B \\
\hline $8 \mathrm{H}-1,130$ & 62.70 & 235.00 & -56.60 & 9.91 E-06 & 90 & A \\
\hline $8 \mathrm{H}-2,130$ & 64.20 & 178.40 & 27.40 & 3.57 E-06 & 80 & B \\
\hline $8 \mathrm{H}-3,130$ & 65.70 & 176.70 & 25.50 & 3.87 E-06 & 80 & B \\
\hline $8 \mathrm{H}-4,130$ & 67.30 & 200.70 & 30.20 & 1.73 E-06 & 70 & B \\
\hline $8 \mathrm{H}-5,130$ & 68.70 & 167.90 & 16.70 & $3.58 \mathrm{E}-06$ & 70 & B \\
\hline $8 \mathrm{H}-6,106$ & 69.60 & 162.20 & 56.80 & $1.03 \mathrm{E}-06$ & 70 & C \\
\hline $9 \mathrm{H}-1,129$ & 72.19 & 269.80 & -76.20 & 7.99 E-06 & 70 & A \\
\hline $9 \mathrm{H}-2,129$ & 73.59 & 184.00 & -21.20 & $3.48 \mathrm{E}-06$ & 70 & A \\
\hline $9 \mathrm{H}-3,128$ & 75.18 & 265.40 & -71.20 & $1.63 \mathrm{E}-06$ & 70 & B \\
\hline $9 \mathrm{H}-4,125$ & 76.65 & 305.60 & 19.10 & $1.95 \mathrm{E}-06$ & 70 & B \\
\hline $9 \mathrm{H}-5,110$ & 78.00 & 295.60 & 23.60 & 2.76 E- 06 & 75 & A \\
\hline $9 \mathrm{H}-6,128$ & 79.68 & 270.90 & -35.80 & 2.79 E- 06 & 75 & A \\
\hline $10 \mathrm{H}-1,129$ & 81.69 & 270.50 & 42.10 & 4.70 E- 06 & 75 & A \\
\hline $10 \mathrm{H}-2,129$ & 83.19 & 280.20 & 42.30 & $2.35 \mathrm{E}-06$ & 75 & A \\
\hline $10 \mathrm{H}-3,128$ & 84.68 & 234.70 & -62.00 & 9.41 E- 07 & 80 & B \\
\hline $10 \mathrm{H}-4,129$ & 86.19 & 290.20 & 19.30 & $1.85 \mathrm{E}-06$ & 80 & A \\
\hline $10 \mathrm{H}-5,131$ & 87.71 & 213.50 & 58.30 & 1.30 E-06 & 80 & B \\
\hline $10 \mathrm{H}-6,131$ & 89.21 & 218.30 & 15.20 & 3.84 E- 07 & 90 & D \\
\hline $10 \mathrm{H}-7,71$ & 90.1 & 1160.50 & 8.70 & $1.63 \mathrm{E}-06$ & 90 & B \\
\hline $11 \mathrm{H}-1,129$ & 91.19 & 263.40 & -59.30 & $3.68 \mathrm{E}-06$ & 60 & A \\
\hline $11 \mathrm{H}-2,129$ & 92.69 & 225.20 & -35.90 & $1.52 \mathrm{E}-06$ & 75 & B \\
\hline $11 \mathrm{H}-3,133$ & 94.23 & 323.30 & -63.40 & $1.99 \mathrm{E}-06$ & 75 & B \\
\hline $11 \mathrm{H}-4,134$ & 95.74 & 135.30 & 28.30 & 1.35 E-06 & 65 & A \\
\hline $11 \mathrm{H}-5,135$ & 97.25 & 194.40 & 69.50 & 3.09 E-06 & 75 & B \\
\hline $11 \mathrm{H}-6,134$ & 98.74 & 200.80 & 30.70 & 3.93 E-06 & 75 & B \\
\hline $12 \mathrm{H}-1,129$ & 100.69 & 177.70 & -54.50 & 4.51 E- 06 & 70 & B \\
\hline $12 \mathrm{H}-2,128$ & 101.97 & 26.20 & 31.70 & 4.09 E- 06 & 70 & A \\
\hline $12 \mathrm{H}-3,130$ & 103.49 & 281.90 & -28.40 & 4.42 E- 06 & 65 & A \\
\hline $12 \mathrm{H}-4,108$ & 104.77 & 254.30 & 50.70 & 3.11 E-06 & 65 & A \\
\hline $12 \mathrm{H}-5,130$ & 106.70 & 231.90 & -32.50 & 8.82 E- 07 & 75 & B \\
\hline $12 \mathrm{H}-6,130$ & 108.20 & 258.60 & 37.40 & 1.77 E-06 & 65 & B \\
\hline $13 \mathrm{H}-1,128$ & 110.18 & 222.70 & -42.80 & $6.81 \mathrm{E}-06$ & 70 & B \\
\hline $13 \mathrm{H}-2,66$ & 111.06 & 201.00 & -60.20 & $4.63 \mathrm{E}-06$ & 70 & A \\
\hline $13 \mathrm{H}-3,70$ & 112.52 & 357.30 & -53.40 & $1.38 \mathrm{E}-06$ & 75 & B \\
\hline $13 \mathrm{H}-4,126$ & 114.66 & 303.80 & -83.30 & 5.75 E- 07 & 75 & B \\
\hline $13 \mathrm{H}-5,75$ & 115.65 & 302.70 & -57.30 & $1.08 \mathrm{E}-06$ & 65 & A \\
\hline $13 \mathrm{H}-6,60$ & 117.00 & 37.20 & -55.20 & $4.10 \mathrm{E}-07$ & 65 & $\mathrm{C}$ \\
\hline $14 \mathrm{H}-1,128$ & 119.68 & 182.50 & -34.00 & $1.98 \mathrm{E}-06$ & 65 & A \\
\hline $14 \mathrm{H}-2,128$ & 121.18 & 187.00 & -35.60 & $1.25 \mathrm{E}-06$ & 65 & A \\
\hline $14 \mathrm{H}-3,128$ & 122.68 & 200.40 & -34.30 & 4.70 E-06 & 65 & A \\
\hline $14 \mathrm{H}-4,128$ & 124.18 & 197.10 & -29.20 & 7.10 E- 07 & 65 & B \\
\hline $14 \mathrm{H}-5,125$ & 125.68 & 195.70 & -27.80 & 3.18 E-06 & 70 & A \\
\hline $14 \mathrm{H}-6,128$ & 127.68 & 133.40 & -18.70 & 4.91 E- 07 & 85 & D \\
\hline $15 \mathrm{H}-1,111$ & 129.01 & 167.50 & 48.40 & 2.93 E- 07 & 85 & D \\
\hline $15 \mathrm{H}-2,134$ & 130.74 & 228.30 & 20.60 & 2.84 E- 07 & 85 & C \\
\hline $15 \mathrm{H}-3,113$ & 132.13 & 96.90 & 31.60 & $2.80 \mathrm{E}-07$ & 60 & B \\
\hline $15 \mathrm{H}-4,128$ & 133.68 & 193.90 & 42.90 & $1.18 \mathrm{E}-06$ & 70 & A \\
\hline $15 \mathrm{H}-6,135$ & 137.38 & 183.90 & -61.20 & 3.61 E-06 & 60 & B \\
\hline $16 \mathrm{H}-1,135$ & 138.78 & 180.20 & 24.10 & 4.70 E- 06 & 65 & C \\
\hline $16 \mathrm{H}-2,135$ & 140.28 & 210.70 & -28.10 & $1.38 \mathrm{E}-06$ & 75 & C \\
\hline $16 \mathrm{H}-3,137$ & 141.70 & 184.60 & -50.60 & 1.42 E-06 & 75 & B \\
\hline $16 \mathrm{H}-4,135$ & 143.37 & 193.70 & -23.40 & $2.26 \mathrm{E}-06$ & 70 & A \\
\hline $16 \mathrm{H}-5,135$ & 144.87 & 181.30 & -76.70 & 1.41 E-06 & 75 & D \\
\hline $16 \mathrm{H}-6,128$ & 146.30 & 191.80 & -40.00 & 1.60 E-06 & 75 & A \\
\hline
\end{tabular}

a Interval is measured from the top of the section.

${ }^{b}$ See Figure 2 for explanation. 
Table 2. Paleomagnetic results of discrete samples from Hole 763A.

\begin{tabular}{|c|c|c|c|c|c|c|}
\hline $\begin{array}{l}\text { Core, section, } \\
\text { interval }(\mathrm{cm})^{\mathrm{a}}\end{array}$ & $\begin{array}{l}\text { Depth } \\
\text { (mbsf) }\end{array}$ & $\begin{array}{c}\text { Declination } \\
\left({ }^{\circ}\right)\end{array}$ & $\begin{array}{l}\text { Inclination } \\
\left({ }^{\circ}\right)\end{array}$ & $\begin{array}{l}\text { NRM } \\
(\mathrm{emu})\end{array}$ & $\begin{array}{l}\text { Peak AF field } \\
(\mathrm{mT})\end{array}$ & $\begin{array}{c}\text { Demagnetization } \\
\text { type }^{b}\end{array}$ \\
\hline \multicolumn{7}{|l|}{$122-763 \mathrm{~A}-$} \\
\hline $1 \mathrm{H}-1,74$ & 0.74 & 30.30 & -35.80 & 1.24 E-07 & 70 & C \\
\hline $1 \mathrm{H}-1,134$ & 1.34 & 2.80 & -49.70 & 1.76 E-06 & 75 & A \\
\hline $1 \mathrm{H}-2,74$ & 2.24 & 331.60 & -49.80 & 1.94 E-06 & 75 & A \\
\hline $1 \mathrm{H}-2,133$ & 2.83 & 316.20 & -60.20 & 3.17 E-06 & 75 & A \\
\hline $1 \mathrm{H}-3,72$ & 3.72 & 350.70 & -13.00 & 7.48 E- 07 & 75 & A \\
\hline $1 \mathrm{H}-3,134$ & 4.34 & 342.60 & -59.90 & 1.75 E- 06 & 75 & A \\
\hline $2 \mathrm{H}-1,70$ & 5.65 & 242.20 & -48.70 & 2.65 E- 06 & 75 & B \\
\hline $2 \mathrm{H}-1,128$ & 6.18 & 274.50 & -48.00 & $3.43 \mathrm{E}-07$ & 75 & A \\
\hline $2 \mathrm{H}-2,66$ & 7.06 & 287.60 & -42.50 & 9.96 E-08 & 75 & B \\
\hline $2 \mathrm{H}-3,66$ & 8.56 & 87.90 & 48.60 & $5.26 \mathrm{E}-08$ & 60 & C \\
\hline $2 \mathrm{H}-3,129$ & 9.19 & 16.40 & -36.00 & 3.21 E- 08 & 85 & D \\
\hline $2 \mathrm{H}-4,64$ & 10.04 & 321.00 & -40.00 & 5.19 E- 08 & 75 & $\mathrm{C}$ \\
\hline $2 \mathrm{H}-4,128$ & 10.68 & 288.30 & -36.70 & 5.77 E- 07 & 65 & A \\
\hline $2 \mathrm{H}-5,46$ & 11.36 & 250.50 & 36.00 & 2.45 E- 08 & 65 & A \\
\hline $2 \mathrm{H}-5,130$ & 12.20 & 296.30 & -43.10 & 5.77 E- 08 & 65 & B \\
\hline $2 \mathrm{H}-6,70$ & 13.10 & 289.80 & -44.70 & 1.92 E-07 & 65 & B \\
\hline $2 \mathrm{H}-6,127$ & 13.68 & 293.20 & -56.00 & $6.28 \mathrm{E}-07$ & 65 & $\mathrm{~A}$ \\
\hline $2 \mathrm{H}-7,22$ & 14.12 & 335.50 & -36.30 & $1.88 \mathrm{E}-07$ & 65 & B \\
\hline $2 \mathrm{H}-7,72$ & 14.62 & 272.20 & -65.60 & 2.31 E-07 & 75 & B \\
\hline $3 \mathrm{H}-1,129$ & 15.69 & 156.70 & -12.50 & 3.65 E-08 & 75 & B \\
\hline $3 \mathrm{H}-2,46$ & 16.36 & 174.50 & -14.70 & 5.67 E- 08 & 75 & B \\
\hline $3 \mathrm{H}-2,130$ & 17.20 & 259.20 & 29.50 & $1.55 \mathrm{E}-08$ & 75 & D \\
\hline $3 \mathrm{H}-3,45$ & 17.85 & 198.80 & 40.70 & $1.05 \mathrm{E}-08$ & 70 & $\mathrm{C}$ \\
\hline $3 \mathrm{H}-3,125$ & 18.66 & 312.70 & 59.50 & $1.88 \mathrm{E}-08$ & 70 & C \\
\hline $3 \mathrm{H}-4,45$ & 19.35 & 337.10 & -13.40 & 2.87 E- 08 & 70 & C \\
\hline $3 \mathrm{H}-4,125$ & 20.15 & 36.60 & -64.40 & 2.66 E- 08 & 70 & C \\
\hline $3 \mathrm{H}-5,44$ & 20.84 & 67.50 & 24.10 & 1.76 E- 08 & 75 & C \\
\hline $3 \mathrm{H}-5,126$ & 21.66 & 228.10 & 64.60 & 9.93 E-09 & 85 & D \\
\hline $3 \mathrm{H}-6,48$ & 22.38 & 267.40 & -42.10 & 3.52 E- 08 & 70 & B \\
\hline $3 \mathrm{H}-6,125$ & 23.15 & 260.50 & 45.10 & 1.42 E- 08 & 90 & D \\
\hline $4 \mathrm{H}-1,36$ & 24.26 & 275.30 & 41.00 & $4.59 \mathrm{E}-08$ & 65 & C \\
\hline $4 \mathrm{H}-1,123$ & 25.13 & 215.00 & 39.60 & $1.29 \mathrm{E}-08$ & 70 & B \\
\hline $4 \mathrm{H}-2,34$ & 25.71 & 97.40 & 6.20 & 4.76 E- 08 & 65 & D \\
\hline $4 \mathrm{H}-2,129$ & 26.69 & 207.20 & 48.50 & 2.63 E-08 & 75 & B \\
\hline $4 \mathrm{H}-3,34$ & 27.21 & 215.70 & 37.80 & 2.80 E- 08 & 75 & D \\
\hline $4 \mathrm{H}-3,130$ & 28.20 & 90.30 & 37.00 & 3.23 E- 08 & 75 & D \\
\hline $4 \mathrm{H}-4,35$ & 28.75 & 240.60 & 28.40 & 2.37 E- 08 & 70 & A \\
\hline $4 \mathrm{H}-4,130$ & 29.43 & 270.20 & 63.80 & 9.06 E-09 & 65 & D \\
\hline $4 \mathrm{H}-5,36$ & 30.26 & 338.00 & 66.40 & 2.12 E- 08 & 65 & D \\
\hline $4 \mathrm{H}-5,130$ & 31.20 & 183.90 & -26.40 & 1.31 E- 08 & 70 & D \\
\hline $4 \mathrm{H}-6,36$ & 31.76 & 182.60 & -62.80 & $1.76 \mathrm{E}-08$ & 70 & D \\
\hline $4 \mathrm{H}-6,130$ & 32.70 & 196.10 & -37.30 & 3.92 E- 08 & 75 & $\mathrm{C}$ \\
\hline $5 \mathrm{H}-1,47$ & 33.87 & 308.70 & 38.20 & $1.99 \mathrm{E}-08$ & 75 & D \\
\hline $5 \mathrm{H}-1,129$ & 34.69 & 50.50 & 38.80 & 1.06 E-08 & 55 & D \\
\hline $5 \mathrm{H}-2,43$ & 35.33 & 125.60 & 34.60 & 1.64 E-08 & 65 & C \\
\hline $5 \mathrm{H}-2,133$ & 36.23 & 216.60 & 61.30 & 9.06 E-09 & 55 & D \\
\hline $5 \mathrm{H}-3,43$ & 36.83 & 343.70 & 22.10 & 3.66 E-08 & 65 & B \\
\hline $5 \mathrm{H}-3,132$ & 37.72 & 242.60 & 64.30 & 9.37 E-09 & 75 & $\mathrm{C}$ \\
\hline $5 \mathrm{H}-4,47$ & 38.37 & 133.20 & 5.90 & 2.47 E- 08 & 55 & C \\
\hline $5 \mathrm{H}-4,132$ & 39.20 & 217.60 & 23.20 & 8.39 E-09 & 75 & D \\
\hline $5 \mathrm{H}-5,45$ & 39.85 & 154.10 & 24.40 & $1.94 \mathrm{E}-08$ & 75 & D \\
\hline $5 \mathrm{H}-5,132$ & 40.72 & 157.70 & 59.50 & 2.12 E-08 & 75 & D \\
\hline $5 \mathrm{H}-6,45$ & 41.35 & 122.30 & 64.80 & $1.33 \mathrm{E}-08$ & 75 & D \\
\hline $5 \mathrm{H}-6,133$ & 42.23 & 268.90 & 35.40 & 1.75 E- 08 & 75 & C \\
\hline $5 \mathrm{H}-7,45$ & 42.85 & 0.80 & 33.90 & 1.92 E- 08 & 75 & $\mathrm{C}$ \\
\hline $6 \mathrm{H}-1,35$ & 43.25 & 29.60 & 54.20 & $2.25 \mathrm{E}-08$ & 75 & D \\
\hline $6 \mathrm{H}-1,125$ & 44.15 & 12.00 & 35.90 & 3.26 E-08 & 75 & $\mathrm{C}$ \\
\hline $6 \mathrm{H}-2,35$ & 44.75 & 221.20 & 48.00 & 2.30 E- 07 & 75 & A \\
\hline $6 \mathrm{H}-2,125$ & 45.65 & 218.70 & -11.40 & 8.45 E- 07 & 80 & A \\
\hline $6 \mathrm{H}-3,35$ & 46.25 & 191.10 & -14.00 & $9.43 \mathrm{E}-07$ & 85 & A \\
\hline $6 \mathrm{H}-3,125$ & 47.15 & 231.50 & 57.20 & $1.68 \mathrm{E}-08$ & 70 & B \\
\hline $6 \mathrm{H}-4,35$ & 47.75 & 127.50 & 59.60 & 8.27 E- 08 & 65 & B \\
\hline $6 \mathrm{H}-4,125$ & 48.65 & 264.50 & 48.90 & 4.27 E-07 & 75 & $\mathrm{C}$ \\
\hline $6 \mathrm{H}-5,35$ & 49.25 & 187.00 & 32.90 & 8.12 E-07 & 65 & $\mathrm{C}$ \\
\hline $6 \mathrm{H}-5,125$ & 50.15 & 206.70 & 44.90 & 1.94 E-06 & 75 & B \\
\hline $6 \mathrm{H}-6,35$ & 50.75 & 36.50 & 54.30 & $1.70 \mathrm{E}-06$ & 80 & B \\
\hline $6 \mathrm{H}-6,125$ & 51.65 & 224.10 & 52.40 & 2.17 E- 06 & 75 & A \\
\hline $6 \mathrm{H}-7,15$ & 52.05 & 204.10 & 42.70 & $1.00 \mathrm{E}-06$ & 75 & $\mathrm{C}$ \\
\hline $6 \mathrm{H}-7,53$ & 52.43 & 2.40 & 28.50 & 7.93 E-07 & 75 & A \\
\hline $7 \mathrm{H}-1,125$ & 53.65 & 250.20 & -55.30 & 2.14 E-06 & 75 & C \\
\hline $7 \mathrm{H}-2,35$ & 54.25 & 97.20 & -52.10 & 2.06 E-06 & 75 & C \\
\hline $7 \mathrm{H}-2,126$ & 55.16 & 117.10 & -48.00 & 2.31 E-06 & 75 & C \\
\hline $7 \mathrm{H}-3,35$ & 55.75 & 302.00 & -45.40 & 1.77 E-06 & 75 & B \\
\hline $7 \mathrm{H}-3,126$ & 56.66 & 125.30 & -72.40 & $2.01 \mathrm{E}-06$ & 75 & A \\
\hline $7 \mathrm{H}-4,35$ & 57.25 & 273.60 & -80.60 & $8.29 \mathrm{E}-07$ & 80 & C \\
\hline $7 \mathrm{H}-4,116$ & 58.06 & 64.50 & -62.60 & $1.74 \mathrm{E}-06$ & 80 & B \\
\hline
\end{tabular}


Table 2 (continued).

\begin{tabular}{|c|c|c|c|c|c|c|}
\hline $\begin{array}{l}\text { Core, section, } \\
\text { interval }(\mathrm{cm})^{\mathrm{a}}\end{array}$ & $\begin{array}{l}\text { Depth } \\
\text { (mbsf) }\end{array}$ & $\begin{array}{c}\text { Declination } \\
\left({ }^{\circ}\right)\end{array}$ & $\begin{array}{l}\text { Inclination } \\
\left({ }^{\circ}\right)\end{array}$ & $\begin{array}{l}\text { NRM } \\
(\mathrm{emu})\end{array}$ & $\begin{array}{l}\text { Peak AF field } \\
\text { (mT) }\end{array}$ & $\begin{array}{c}\text { Demagnetization } \\
\text { type }^{b}\end{array}$ \\
\hline $7 \mathrm{H}-5,35$ & 58.75 & 88.90 & -28.20 & 4.32 E-06 & 80 & B \\
\hline $7 \mathrm{H}-5,126$ & 59.66 & 240.90 & -27.90 & 3.42 E-06 & 80 & C \\
\hline $7 \mathrm{H}-6,35$ & 60.25 & 20.50 & -28.80 & $1.61 \mathrm{E}-06$ & 80 & C \\
\hline $7 H-6,126$ & 61.16 & 121.30 & -72.40 & 7.42 E-06 & 80 & B \\
\hline $8 \mathrm{H}-1,35$ & 62.25 & 91.50 & -76.70 & $4.58 \mathrm{E}-06$ & 80 & B \\
\hline $8 \mathrm{H}-1,125$ & 63.15 & 134.80 & -15.30 & .2 .54 E-06 & 70 & B \\
\hline $8 \mathrm{H}-2,35$ & 63.75 & 95.30 & -26.70 & 1.12 E-06 & 70 & C \\
\hline $8 \mathrm{H}-2,127$ & 64.67 & 77.50 & 51.50 & 6.43 E-07 & 70 & D \\
\hline $8 \mathrm{H}-3,35$ & 65.25 & 159.90 & 22.00 & $1.85 \mathrm{E}-06$ & 70 & B \\
\hline $8 \mathrm{H}-3,126$ & 66.16 & 120.70 & -31.40 & $3.43 \mathrm{E}-06$ & 70 & A \\
\hline $8 \mathrm{H}-4,35$ & 66.75 & 105.80 & -46.70 & 2.44 E-06 & 70 & A \\
\hline $8 \mathrm{H}-4,126$ & 67.66 & 190.10 & 17.50 & $1.16 \mathrm{E}-06$ & 70 & C \\
\hline $8 \mathrm{H}-5,35$ & 68.25 & 109.20 & 18.50 & $3.56 \mathrm{E}-06$ & 70 & B \\
\hline $8 \mathrm{H}-5,126$ & 69.16 & 103.20 & -39.70 & 1.70 E-06 & 70 & A \\
\hline $8 \mathrm{H}-6,35$ & 69.75 & 63.60 & -11.20 & 2.73 E- 06 & 70 & B \\
\hline $8 \mathrm{H}-6,126$ & 70.66 & 84.80 & -13.20 & 2.03 E-06 & 70 & C \\
\hline $8 \mathrm{H}-7,35$ & 71.25 & 73.20 & -58.30 & 5.57 E-06 & 70 & A \\
\hline $8 \mathrm{H}-7,75$ & 71.65 & 132.80 & -49.30 & 3.63 E-06 & 65 & A \\
\hline $9 \mathrm{H}-1,70$ & 72.10 & 81.00 & -64.70 & 9.46 E- 06 & 60 & A \\
\hline $9 \mathrm{H}-1,125$ & 72.65 & 312.80 & -74.40 & $1.88 \mathrm{E}-06$ & 60 & A \\
\hline $9 \mathrm{H}-2,35$ & 73.25 & 43.00 & -26.30 & 2.05 E-06 & 65 & A \\
\hline $9 \mathrm{H}-2,125$ & 74.15 & 96.90 & -59.50 & $1.16 \mathrm{E}-06$ & 75 & B \\
\hline $9 \mathrm{H}-3,35$ & 74.75 & 89.50 & -32.40 & 1.99 E-06 & 75 & B \\
\hline $9 \mathrm{H}-3,125$ & 75.65 & 168.10 & -45.60 & 2.57 E-06 & 60 & A \\
\hline $9 \mathrm{H}-4,35$ & 76.25 & 122.10 & -41.20 & 1.41 E-06 & 60 & A \\
\hline $9 \mathrm{H}-4,125$ & 77.15 & 152.60 & -30.60 & 1.74 E-06 & 65 & A \\
\hline $9 \mathrm{H}-5,35$ & 77.75 & 68.40 & -21.10 & 1.34 E-06 & 65 & B \\
\hline $9 \mathrm{H}-5,125$ & 78.65 & 241.30 & -23.80 & $1.48 \mathrm{E}-06$ & 65 & B \\
\hline $9 \mathrm{H}-6,35$ & 79.25 & 31.20 & -10.90 & 1.41 E-06 & 65 & B \\
\hline $9 \mathrm{H}-6,125$ & 80.15 & 154.50 & -63.40 & 2.10 E-06 & 60 & A \\
\hline $9 \mathrm{H}-7,20$ & 80.60 & 333.10 & 56.80 & 1.33 E- 06 & 60 & A \\
\hline $10 \mathrm{H}-1,50$ & 81.40 & 137.50 & 11.50 & 1.86 E- 06 & 60 & A \\
\hline $10 \mathrm{H}-1,126$ & 82.16 & 150.10 & 33.20 & 1.76 E- 06 & 60 & B \\
\hline $10 \mathrm{H}-2,36$ & 82.76 & 181.70 & 30.90 & 6.95 E-07 & 60 & B \\
\hline $10 \mathrm{H}-2,125$ & 83.65 & 149.70 & 24.80 & 1.40 E-06 & 65 & B \\
\hline $10 \mathrm{H}-3,35$ & 84.25 & 184.60 & 17.60 & $1.43 \mathrm{E}-06$ & 65 & B \\
\hline $10 \mathrm{H}-3,124$ & 85.14 & 77.00 & 20.70 & 1.02 E-06 & 65 & B \\
\hline $10 \mathrm{H}-4,35$ & 85.75 & 145.40 & 28.70 & 2.40 E- 06 & 75 & A \\
\hline $10 \mathrm{H}-4,115$ & 86.55 & 163.20 & 20.20 & 2.01 E-06 & 70 & A \\
\hline $10 \mathrm{H}-5,35$ & 87.25 & 134.70 & 39.00 & 3.36 E-06 & 70 & A \\
\hline $10 \mathrm{H}-5,125$ & 88.15 & 187.70 & 3.80 & 2.43 E- 06 & 70 & B \\
\hline $10 \mathrm{H}-6,35$ & 88.75 & 114.50 & 36.20 & 2.93 E-06 & 70 & A \\
\hline $10 \mathrm{H}-6,125$ & 89.65 & 175.30 & 2.80 & 4.78 E- 06 & 70 & A \\
\hline $10 \mathrm{H}-7,52$ & 90.42 & 99.90 & -1.10 & 3.14 E-06 & 70 & B \\
\hline $11 \mathrm{H}-2,90$ & 93.00 & 136.60 & -63.40 & $3.19 \mathrm{E}-06$ & 70 & C \\
\hline $11 \mathrm{H}-3,34$ & 93.74 & 161.90 & 20.30 & 6.95 E-07 & 65 & A \\
\hline $11 \mathrm{H}-3,120$ & 94.40 & 164.10 & -68.40 & 2.65 E-06 & 65 & B \\
\hline $11 \mathrm{H}-4,34$ & 95.25 & 141.90 & 12.60 & 3.57 E-06 & 65 & B \\
\hline $11 \mathrm{H}-4,120$ & 95.90 & 146.10 & 6.50 & $3.63 \mathrm{E}-06$ & 65 & C \\
\hline $11 \mathrm{H}-5,34$ & 96.74 & 118.60 & 24.40 & 9.39 E-07 & 65 & B \\
\hline $11 \mathrm{H}-5,120$ & 97.40 & 161.20 & 5.40 & $3.60 \mathrm{E}-06$ & 65 & B \\
\hline $11 \mathrm{H}-6,34$ & 98.25 & 119.60 & 18.60 & $1.13 \mathrm{E}-06$ & 60 & B \\
\hline $11 \mathrm{H}-6,120$ & 98.90 & 165.60 & -2.50 & $3.81 \mathrm{E}-06$ & 60 & C \\
\hline $11 \mathrm{H}-7,35$ & 99.75 & 64.30 & -14.60 & 2.72 E-06 & 60 & B \\
\hline $12 \mathrm{H}-1,34$ & 100.24 & 162.80 & -51.40 & 1.90 E-06 & 75 & A \\
\hline $12 \mathrm{H}-1,124$ & 101.14 & 195.60 & 32.60 & $1.22 \mathrm{E}-06$ & 70 & B \\
\hline $12 \mathrm{H}-2,34$ & 101.74 & 179.10 & 15.50 & 9.97 E-07 & 75 & B \\
\hline $12 \mathrm{H}-2,124$ & 102.64 & 188.40 & 10.00 & $1.78 \mathrm{E}-06$ & 75 & C \\
\hline $12 \mathrm{H}-3,34$ & 103.24 & 125.80 & -11.90 & 2.33 E-07 & 80 & C \\
\hline $12 \mathrm{H}-3,124$ & 104.14 & 171.90 & -48.90 & 2.27 E-06 & 80 & B \\
\hline $12 \mathrm{H}-4,34$ & 104.74 & 151.60 & -46.20 & 3.13 E-06 & 80 & A \\
\hline $12 \mathrm{H}-4,125$ & 105.65 & 221.10 & 27.10 & $1.13 \mathrm{E}-06$ & 70 & B \\
\hline $12 \mathrm{H}-5,34$ & 106.24 & 102.50 & 46.90 & 2.53 E-06 & 70 & A \\
\hline $12 \mathrm{H}-5,124$ & 107.14 & 166.40 & -6.70 & 2.46 E-06 & 70 & B \\
\hline $12 \mathrm{H}-6,34$ & 107.74 & 74.20 & -34.20 & $1.80 \mathrm{E}-06$ & 70 & $\mathrm{~A}$ \\
\hline $12 \mathrm{H}-6,124$ & 108.64 & 182.20 & 31.10 & $4.00 \mathrm{E}-06$ & 70 & A \\
\hline $12 \mathrm{H}-7,34$ & 109.24 & 11.90 & 70.20 & 1.75 E-06 & 65 & B \\
\hline $12 \mathrm{H}-7,67$ & 109.57 & 121.00 & 25.20 & 3.07 E-06 & 65 & A \\
\hline $13 \mathrm{H}-1,77$ & 110.17 & 189.40 & -29.50 & 2.66 E-06 & 65 & A \\
\hline $13 \mathrm{H}-1,125$ & 110.65 & 322.90 & -56.00 & 3.45 E-06 & 65 & B \\
\hline $13 \mathrm{H}-2,77$ & 111.67 & 255.80 & -69.00 & $2.22 \mathrm{E}-06$ & 65 & B \\
\hline $13 \mathrm{H}-2,127$ & 112.17 & 211.90 & -49.90 & $2.78 \mathrm{E}-06$ & 60 & A \\
\hline $13 \mathrm{H}-3,78$ & 113.18 & 275.80 & 53.70 & 8.76 E-07 & 60 & C \\
\hline $13 \mathrm{H}-3,127$ & 113.67 & 294.30 & 23.10 & 3.12 E-06 & 60 & A \\
\hline $13 \mathrm{H}-4,76$ & 114.66 & 220.90 & 43.90 & 5.61 E-07 & 70 & D \\
\hline $13 \mathrm{H}-4,105$ & 114.95 & 166.90 & -17.90 & 2.07 E-06 & 70 & B \\
\hline $13 \mathrm{H}-5,78$ & 116.18 & 163.30 & -23.10 & $1.65 \mathrm{E}-06$ & 70 & B \\
\hline
\end{tabular}


Table 2 (continued),

\begin{tabular}{|c|c|c|c|c|c|c|}
\hline $\begin{array}{l}\text { Core, section, } \\
\text { interval }(\mathrm{cm})^{\mathrm{a}}\end{array}$ & $\begin{array}{l}\text { Depth } \\
\text { (mbsf) }\end{array}$ & $\begin{array}{l}\text { Declination } \\
\left({ }^{\circ}\right)\end{array}$ & $\begin{array}{c}\text { Inclination } \\
\left({ }^{\circ}\right)\end{array}$ & $\begin{array}{l}\text { NRM } \\
\text { (emu) }\end{array}$ & $\begin{array}{l}\text { Peak AF field } \\
\text { (mT) }\end{array}$ & $\begin{array}{l}\text { Demagnetization } \\
\text { type }^{b}\end{array}$ \\
\hline $13 \mathrm{H}-5,127$ & 116.67 & 196.70 & -24.80 & 8.07 E-07 & 70 & A \\
\hline $13 \mathrm{H}-6,68$ & 117.58 & 124.70 & -23.90 & $2.06 \mathrm{E}-06$ & 70 & B \\
\hline $13 \mathrm{H}-6,127$ & 118.17 & 105.30 & -37.80 & $1.63 \mathrm{E}-06$ & 70 & B \\
\hline $13 \mathrm{H}-7,46$ & 118.86 & 58.40 & -13.30 & $2.21 \mathrm{E}-06$ & 70 & B \\
\hline $14 \mathrm{H}-1,80$ & 119.70 & 289.70 & -52.00 & $9.59 \mathrm{E}-07$ & 70 & C \\
\hline $14 \mathrm{H}-1,128$ & 120.18 & 270.00 & 21.00 & $1.18 \mathrm{E}-07$ & 70 & B \\
\hline $14 \mathrm{H}-2,78$ & 121.18 & 242.40 & 43.40 & $6.06 \mathrm{E}-07$ & 70 & C \\
\hline $14 \mathrm{H}-2,129$ & 121.69 & 315.50 & 30.40 & 8.21 E- 07 & 70 & B \\
\hline $14 \mathrm{H}-3,79$ & 122.69 & 154.20 & 66.40 & 6.39 E-07 & 70 & C \\
\hline $14 \mathrm{H}-3,129$ & 123.19 & 276.50 & 71.00 & 8.35 E- 07 & 70 & C \\
\hline $14 \mathrm{H}-4,48$ & 123.88 & 110.20 & 43.30 & $7.02 \mathrm{E}-07$ & 70 & C \\
\hline $14 \mathrm{H}-4,127$ & 124.67 & 318.00 & 60.70 & $1.84 \mathrm{E}-06$ & 70 & A \\
\hline $14 \mathrm{H}-5,48$ & 125.38 & 113.30 & 51.50 & $1.16 \mathrm{E}-06$ & 70 & A \\
\hline $14 \mathrm{H}-5,129$ & 126.19 & 67.50 & 72.50 & $1.06 \mathrm{E}-06$ & 70 & B \\
\hline $14 \mathrm{H}-6,49$ & 126.89 & 84.90 & -31.90 & $1.07 \mathrm{E}-06$ & 70 & C \\
\hline $14 \mathrm{H}-6,129$ & 127.69 & 171.90 & 67.80 & $1.23 \mathrm{E}-06$ & 70 & C \\
\hline $14 \mathrm{H}-7,49$ & 128.39 & 66.60 & 53.60 & $1.69 \mathrm{E}-06$ & 70 & A \\
\hline $15 \mathrm{H}-1,45$ & 128.85 & 349.40 & -43.90 & 6.23 E- 07 & 70 & A \\
\hline $15 \mathrm{H}-1,132$ & 129.72 & 315.00 & -19.20 & $1.79 \mathrm{E}-07$ & 70 & D \\
\hline $15 \mathrm{H}-2,45$ & 130.35 & 308.50 & 1.80 & $8.59 \mathrm{E}-07$ & 70 & B \\
\hline $15 \mathrm{H}-2,130$ & 131.20 & 322.40 & 14.50 & 4.32 E- 07 & 70 & B \\
\hline $15 \mathrm{H}-3,45$ & 131.85 & 347.80 & 24.50 & $5.34 \mathrm{E}-07$ & 70 & A \\
\hline $15 \mathrm{H}-3,130$ & 132.70 & 259.40 & 29.60 & $1.95 \mathrm{E}-07$ & 70 & B \\
\hline $15 \mathrm{H}-4,43$ & 133.33 & 168.30 & -54.10 & $3.09 \mathrm{E}-07$ & 70 & C \\
\hline $15 \mathrm{H}-4,133$ & 134.23 & 247.90 & 48.70 & $2.09 \mathrm{E}-06$ & 70 & A \\
\hline $15 \mathrm{H}-5,47$ & 134.87 & 276.80 & 58.70 & $1.23 \mathrm{E}-06$ & 70 & B \\
\hline $15 \mathrm{H}-5,133$ & 135.73 & 164.30 & -43.90 & $1.10 \mathrm{E}-06$ & 70 & B \\
\hline $15 \mathrm{H}-6,46$ & 136.36 & 87.50 & -42.60 & $3.47 \mathrm{E}-06$ & 70 & A \\
\hline $15 \mathrm{H}-6,135$ & 137.25 & 147.40 & -13.20 & $2.14 \mathrm{E}-06$ & 70 & A \\
\hline $15 \mathrm{H}-7,47$ & 137.87 & 43.80 & -43.60 & $1.64 \mathrm{E}-06$ & 70 & B \\
\hline $16 \mathrm{H}-1,44$ & 138.34 & 311.40 & -62.40 & $2.07 \mathrm{E}-06$ & 70 & B \\
\hline $16 \mathrm{H}-1,127$ & 139.17 & 187.80 & 28.70 & $1.43 \mathrm{E}-06$ & 70 & B \\
\hline $16 \mathrm{H}-2,44$ & 139.84 & 105.50 & 69.20 & $1.07 \mathrm{E}-06$ & 70 & B \\
\hline $16 \mathrm{H}-2,128$ & 140.68 & 167.70 & 34.00 & $1.57 \mathrm{E}-06$ & 70 & B \\
\hline $16 \mathrm{H}-3,45$ & 141.35 & 132.90 & 54.00 & $6.33 \mathrm{E}-07$ & 70 & C \\
\hline $16 \mathrm{H}-3,128$ & 142.18 & 166.90 & 23.50 & $1.53 \mathrm{E}-06$ & 70 & B \\
\hline $16 \mathrm{H}-4,45$ & 142.85 & 161.20 & 32.70 & $2.42 \mathrm{E}-06$ & 70 & A \\
\hline $16 \mathrm{H}-4,114$ & 143.54 & 156.80 & 24.80 & $2.08 \mathrm{E}-06$ & 70 & B \\
\hline $16 \mathrm{H}-5,46$ & 144.36 & 131.60 & 28.70 & 9.04 E-07 & 70 & C \\
\hline $16 \mathrm{H}-5,144$ & 145.34 & 160.60 & 52.40 & $7.78 \mathrm{E}-07$ & 70 & A \\
\hline $16 \mathrm{H}-6,53$ & 145.93 & 103.50 & 27.00 & 5.59 E- 07 & 70 & A \\
\hline $16 \mathrm{H}-6,133$ & 146.73 & 156.00 & 15.50 & $9.69 \mathrm{E}-07$ & 70 & B \\
\hline $16 \mathrm{H}-7,23$ & 147.13 & 69.50 & 76.50 & 8.67 E- 07 & 70 & A \\
\hline $17 \mathrm{H}-1,24$ & 147.64 & 198.70 & -34.10 & 8.32 E- 07 & 70 & B \\
\hline $17 \mathrm{H}-1,130$ & 148.70 & 193.30 & -38.90 & $1.10 \mathrm{E}-06$ & 70 & B \\
\hline $17 \mathrm{H}-2,25$ & 149.15 & 201.90 & 0.80 & 7.79 E-07 & 70 & A \\
\hline $17 \mathrm{H}-2,112$ & 150.02 & 217.00 & 1.40 & 3.17 E-07 & 70 & D \\
\hline $17 \mathrm{H}-3,52$ & 150.92 & 152.40 & 25.20 & $1.80 \mathrm{E}-06$ & 70 & B \\
\hline $17 \mathrm{H}-3,104$ & 151.42 & 168.20 & 12.30 & $5.91 \mathrm{E}-06$ & 60 & C \\
\hline $17 \mathrm{H}-4,54$ & 152.44 & 38.00 & -18.00 & $1.10 \mathrm{E}-06$ & 60 & A \\
\hline $17 \mathrm{H}-4,132$ & 153.22 & 81.80 & 61.70 & $1.53 \mathrm{E}-06$ & 60 & C \\
\hline $17 \mathrm{H}-5,54$ & 153.94 & 148.20 & -10.50 & $4.10 \mathrm{E}-06$ & 60 & B \\
\hline $17 \mathrm{H}-5,121$ & 154.61 & 172.60 & 38.50 & $1.39 \mathrm{E}-06$ & 60 & C \\
\hline $17 \mathrm{H}-6,27$ & 155.17 & 78.00 & 51.70 & 3.37 E-06 & 60 & A \\
\hline $17 \mathrm{H}-6,127$ & 156.17 & 167.00 & 20.40 & $2.15 \mathrm{E}-06$ & 60 & C \\
\hline $17 \mathrm{H}-7,21$ & 156.61 & 123.70 & 60.80 & $3.20 \mathrm{E}-06$ & 60 & A \\
\hline $18 \mathrm{H}-1,51$ & 157.41 & 130.20 & -35.00 & 6.47 E-07 & 60 & C \\
\hline $18 \mathrm{H}-1,134$ & 158.24 & 336.10 & 45.00 & $7.06 \mathrm{E}-07$ & 60 & C \\
\hline $18 \mathrm{H}-2,50$ & 158.90 & 176.60 & 15.60 & $1.73 \mathrm{E}-06$ & 80 & A \\
\hline $18 \mathrm{H}-2,131$ & 159.71 & 139.90 & -35.80 & $1.68 \mathrm{E}-06$ & 80 & A \\
\hline
\end{tabular}

a Interval is measured from the top of the section.

${ }^{\mathrm{b}}$ See Figure 2 for explanation.

at 25.2 mbsf (Fig. 5 and Table 3). Below $100.1 \mathrm{mbsf}$, the reversal stratigraphy becomes uninterpretable due to the presence of three hiatuses at 112, 122, and 132 mbsf (see "Biostratigraphy" section, "Site 762"' chapter, Haq, von Rad, O'Connell, et al., 1990).

\section{Hole 763 A}

Reliable paleomagnetic results (Table 2 and Fig. 4) were obtained from Hole 763A. All major polarity reversals from 18 Ma to the present (Table 3) were observed. The reversal stratigraphy is based primarily on the inclination data from discrete samples because of disagreement between paleomagnetic results obtained from shipboard measurements and discrete samples. The discrepancy is probably due to the inability of removing secondary overprints with $9 \mathrm{mT}$ alternating field. Above $141 \mathrm{mbsf}$, polarity assignments are less ambiguous and can be correlated to the geomagnetic polarity time scale, from Chron 1 to Chron 4A (Fig. 5).

\section{Downhole Fluctuations of NRM Intensity and Susceptibility}

Hole $762 B$

Figure 3 also shows the whole-core susceptibility, the NRM intensity, and intensity after 9-mT demagnetization of archive sections plotted against depth. Although the demag- 

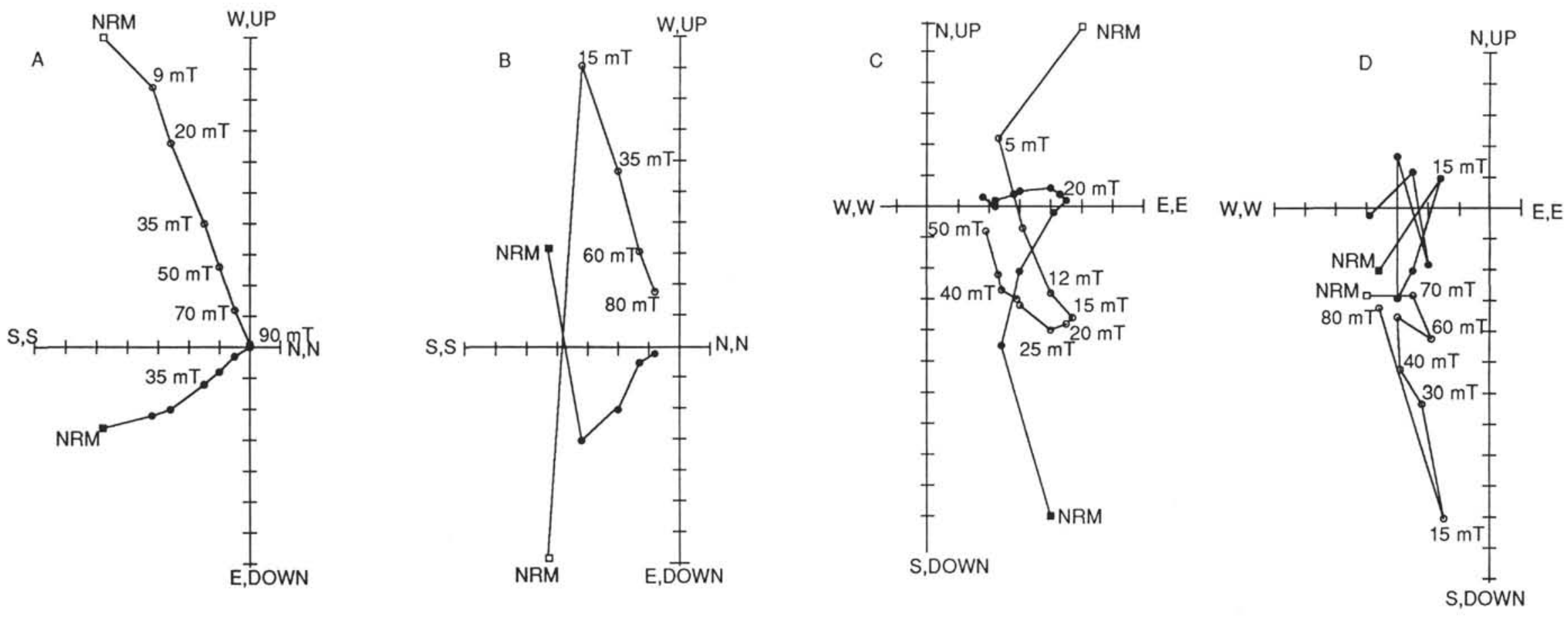

- Vertical Component

- Horizontal Component

Figure 2. Zijderveld orthogonal projection diagrams of discrete samples from Holes 762B and 763A subjected to alternating field demagnetization. A. Sample $122-762 \mathrm{~B}-7 \mathrm{H}-5$, $130-132 \mathrm{~cm}$ B. Sample 122-763A-11H-3, 120-122 cm. C. Sample 122-763A-2H-3, 66-68 cm. D. Sample 122-763A-4H-4, 130-132 cm. 


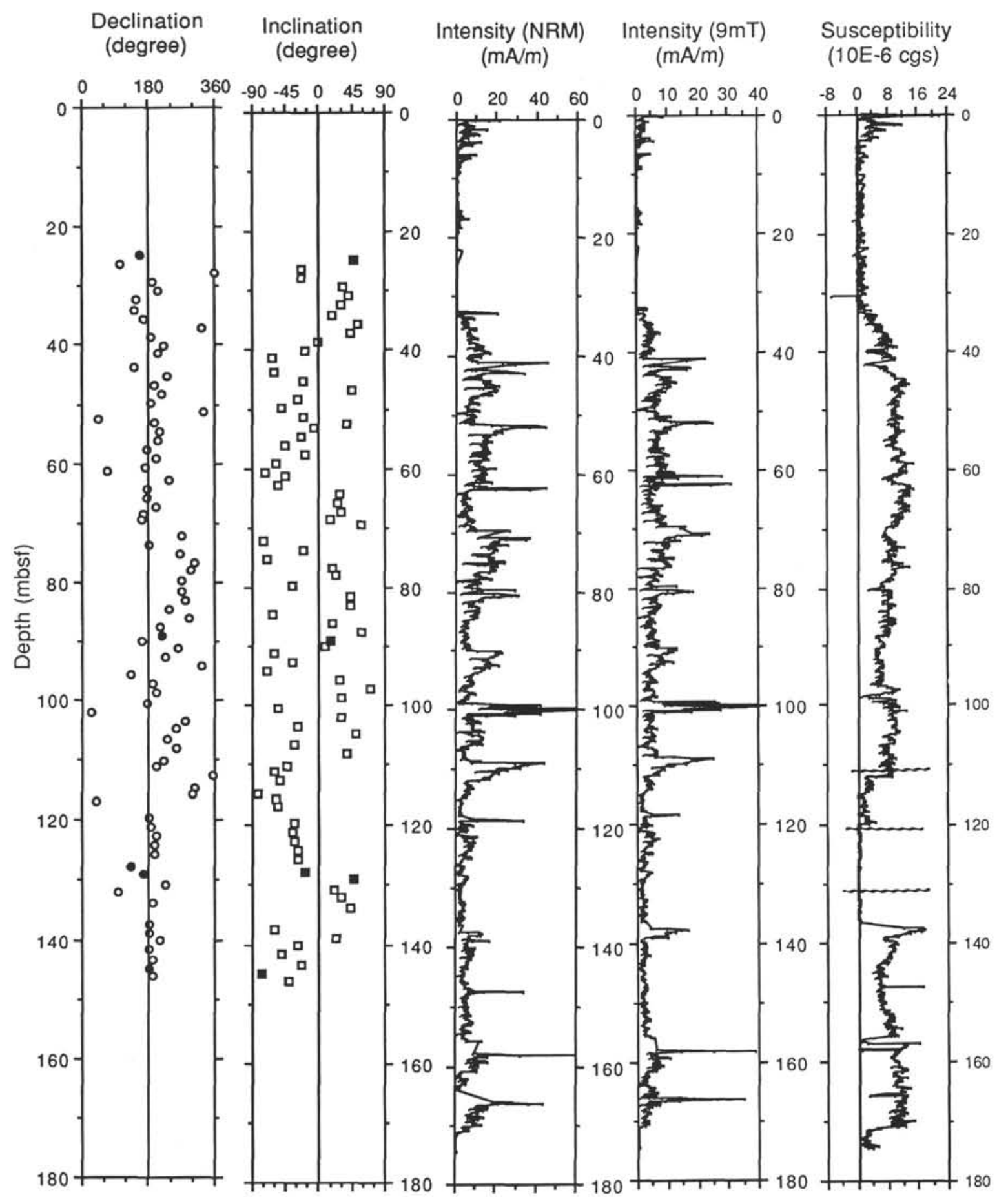

Figure 3. Inclination and relative declination of discrete samples and whole-core NRM intensity, intensity after 9-mT demagnetization, and susceptibility as a function of depth in Hole 762B. Solid circles and squares represent the samples of demagnetization type D (Fig. 2). Wavy lines represent the hiatuses seen from biostratigraphic data.

netization in 9-mT AF reduces the NRM intensity by approximately $37.5 \%$, the main features of the NRM intensity remain unchanged. The spikes of the NRM intensity shown in Figure 3 are considered to be rust contamination from the core catcher during coring because their positions can be correlated precisely to the bottom of each core. Despite the presence of these spikes, a relatively high value of the NRM intensity can still be seen in the interval 33-115 mbsf. Whole- core susceptibility shown in Figure 3 also displays similar downhole fluctuations.

Comparing the magnetic results with lithology (see "Lithostratigraphy" section, "Site 762"' chapter, Haq, von Rad, O'Connell, et al., 1990) and geochemistry (De Carlo, this volume) from this hole, the NRM intensity and the susceptibility profiles appear to correlate to lithologic units and fluctuations in iron content. For instance, Subunit IA 


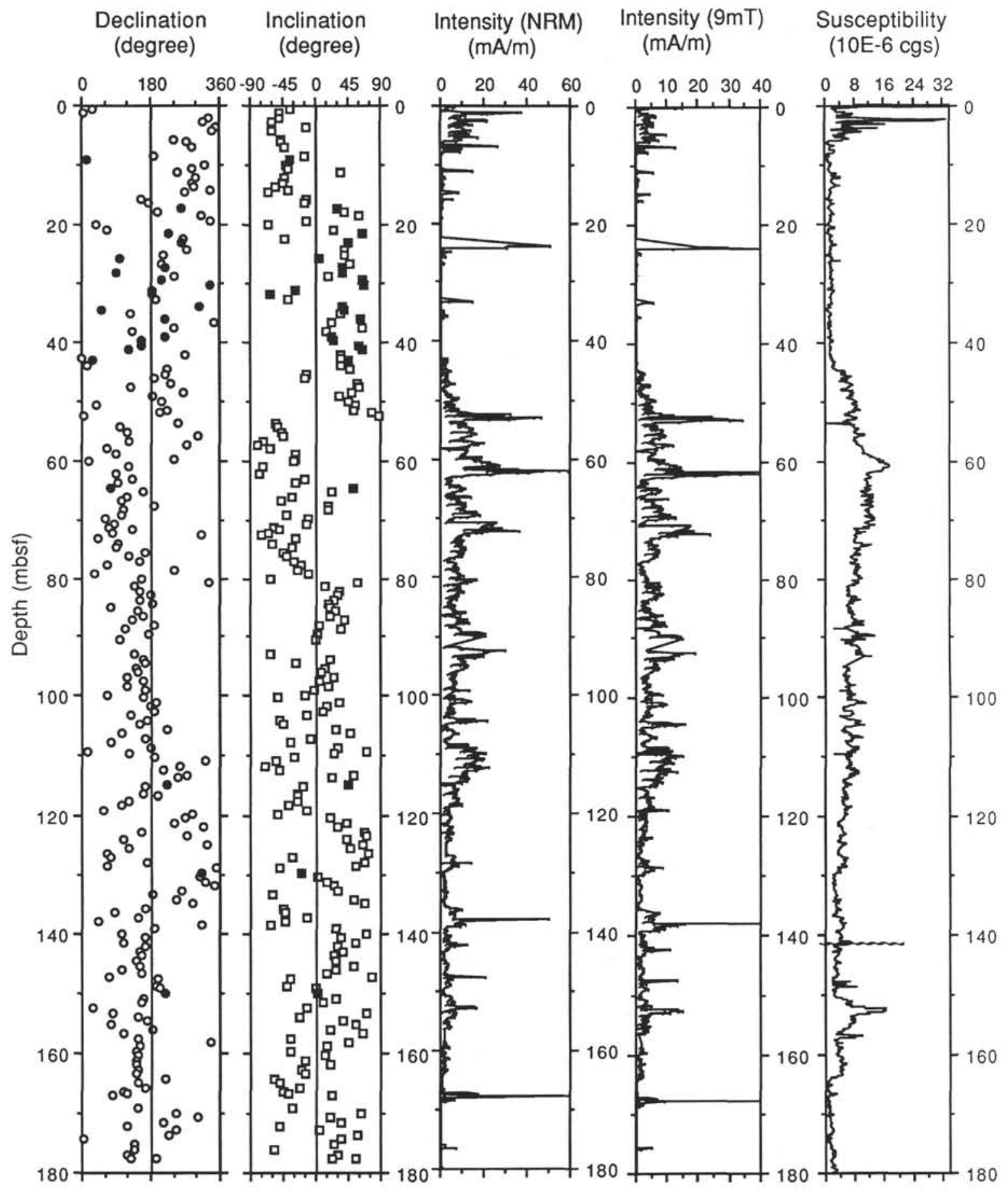

Figure 4. Inclination and relative declination of discrete samples and whole-core NRM intensity, intensity after 9-mT demagnetization, and susceptibility as a function of depth in Hole 763A. Solid circles and squares represent the samples of demagnetization type D (Fig. 2). Wavy lines represent the hiatuses seen from biostratigraphic data.

(0-61.4 mbsf) is represented mainly by foraminifer-nannofossil ooze with a small amount of terrigenous material (e.g., clay minerals). The NRM intensity and the susceptibility profiles show an extremely low value in the interval 5-37 mbsf and relatively high value with fluctuations in the interval 37-61.4 mbsf. The high-value interval correlates to increased clay content (iron content around 0.12 parts per million, or ppm) and the low-value interval to dominant foraminifer and low iron content $(\sim 0.03 \mathrm{ppm})$. Within Sub- unit IB (61.4-118.4 mbsf) the NRM intensity and susceptibility values stay relatively high with a maximum at the top and bottom of the subunit. Similarly, the high values of susceptibility correlate well to the occurrence of terrigenous material (e.g., mica, clay, and accessory minerals) from the lithology and higher iron content $(\sim 0.38 \mathrm{ppm})$ from the geochemistry. Furthermore, a positive correlation between iron and aluminum content is found in the geochemistry study (De Carlo, this volume), where aluminum is an index 


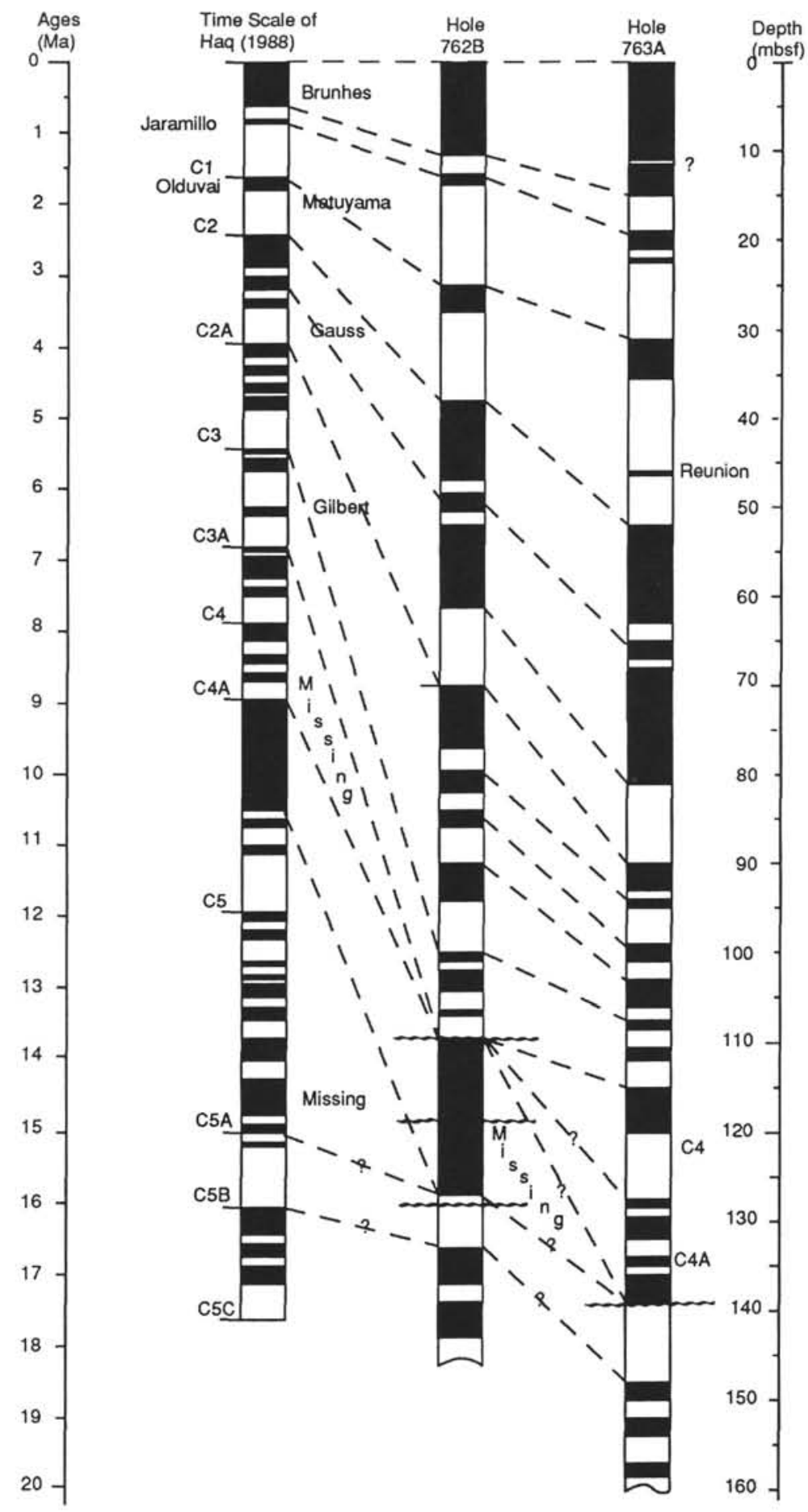

Figure 5. Correlations of the magnetostratigraphy of Holes 762B and 763A with the magnetic time scale of Haq et al. (1988) and the age of the Reunion event from Berggren et al. (1985). Wavy lines represent the hiatuses seen from biostratigraphic data.

of clay content (Kennett, 1982). Therefore, changes in depositional processes seem to control the downhole variation of magnetic record.

Below 118.4 mbsf, the color of the lithology changes from light grey to white and pale yellow until 127.9 mbsf. Extremely low values of susceptibility in this interval correspond to the maximum of carbonate content $\left(\sim 90 \% \mathrm{CaCO}_{3}\right)$. The correlation between the downhole fluctuations of magnetic record and the lithology (especially clay content) suggests the different sediment transport mechanisms that allow terrigenous material to be deposited.

\section{Hole $763 A$}

Figure 4 also shows the whole-core susceptibility, NRM intensity, and intensity after 9-mT demagnetization of archive sections plotted against depth. For the same reason, spikes of 
Table 3. Magnetic polarity reversal boundaries identified in APC cores from Holes 762B and 763A.

\begin{tabular}{|c|c|c|c|}
\hline \multirow{2}{*}{$\begin{array}{l}\text { Age } \\
\text { (Ma) }\end{array}$} & \multicolumn{2}{|c|}{ Depth (mbsf) } & \multirow[b]{2}{*}{ Boundary or event } \\
\hline & Hole 762B & Hole $763 \mathrm{~A}$ & \\
\hline 0.73 & 11.20 & 15.10 & $\begin{array}{l}\text { Brunhes/ } \\
\text { Matuyama }\end{array}$ \\
\hline 0.91 & 12.10 & 18.30 & upper Jaramillo \\
\hline 0.98 & 13.10 & 21.10 & lower Jaramillo \\
\hline 1.65 & 25.00 & 31.10 & upper Olduvai \\
\hline 1.88 & 27.50 & 35.70 & lower Olduvai \\
\hline 2.01 & & 45.80 & Reunion? \\
\hline 2.50 & 37.60 & 52.30 & Matuyama/Gauss \\
\hline 2.92 & 47.20 & 64.20 & Kaena \\
\hline 3.08 & 51.40 & 67.80 & Mammoth \\
\hline 3.40 & 62.10 & 81.50 & Gauss/Gilbert \\
\hline 3.90 & 71.60 & 90.30 & Cochiti \\
\hline 4.10 & 79.50 & 90.10 & Nunivak \\
\hline 4.41 & 84.50 & 94.50 & Sidufjall \\
\hline 4.57 & 90.00 & 99.50 & Thvera \\
\hline 5.40 & 100.10 & 107.90 & Base of Gilbert \\
\hline 6.70 & 110.40 & 115.80 & Base of $\mathrm{C} 3 \mathrm{~A}$ ? \\
\hline 7.80 & & 127.60 & Base of $\mathrm{C} 4$ ? \\
\hline 8.95 & & 139.60 & Base of C4A? \\
\hline 11.70 & 126.20 & & Base of C5? \\
\hline 16.10 & 134.30 & 147.10 & Base of C5B? \\
\hline
\end{tabular}

NRM intensity can be considered to be rust contamination from the core catcher. The variation in NRM intensity parallels the variation seen in the susceptibility measurement and can be correlated well to terrigenous material.

In general, susceptibility values are low (mostly between 1 and $15 \times 10^{-6} \mathrm{cgs}$ ), with the exception of a rust-contaminated top section ( $0-4 \mathrm{mbsf})$ that yielded susceptibility values in excess of $3.5 \times 10^{-5} \mathrm{cgs}$. The interval between 15 and $40 \mathrm{mbsf}$ corresponds to a section of white or light gray sediment (foraminifer nannofossil oozes with clay) of early Quaternary age. The color of this sediment suggests a low concentration of magnetic material (iron content around $0.08 \mathrm{ppm}$ ), which is consistent with the extremely low values of NRM intensity (around $0.1 \mathrm{~mA} / \mathrm{m}$ ) and susceptibility (around $0.5-3 \times 10^{-6}$ cgs). Below this interval to a depth of $110 \mathrm{mbsf}$, susceptibility and NRM intensity values are generally higher than elsewhere in the profile (i.e., between 5 and $15 \times 10^{-6} \mathrm{cgs}, 1-8 \mathrm{~mA} / \mathrm{m}$, respectively), which corresponds to color changes from light gray to light greenish brown and higher iron content $(\sim 0.55$ ppm). This correspondence is particularly clear in the interval from 150 to 155 mbsf, which is characterized by olive yellow to pale yellow sediment (clayey foraminifer-nannofossil oozes with chalk) and a high content of clay and quartz (see "Lithostratigraphy" section, "Site 763" chapter, Haq, von Rad, O'Connell, et al., 1990). The clear lithologic control of magnetic susceptibility variations in Holes 762B and 763A provides a potential basis for a correlation between Hole 762 B and $763 \mathrm{~A}$.

\section{Correlation Between Holes 762B and 763A}

The high-resolution magnetic susceptibility profiles obtained from Holes 762B and 763A (Figs. 3 and 4) yield a good correlation between these holes. It is extremely difficult to make visual identification of fine-scale lithostratigraphic correlation when the susceptibility profiles are plotted on the scales used in Figure 6. Therefore, I have replotted selected portions from each profile on the expanded scales in Figure 7 in order to better illustrate the extent of correlation.

Correlation between the susceptibility profiles of each hole is based on the relative position, intensity, and configuration of first-order peaks $\left(20-40 \times 10^{-6} \mathrm{cgs}\right)$ and second-order peaks $\left(5-10 \times 10^{-6} \mathrm{cgs}\right)$. Figures 6 and 7 show possible correlations between the two holes. The reliability of specific correlations is variable. Susceptibility values of less than $1 \times 10^{-6} \mathrm{cgs}$ are close to the limit of detection of the whole-core sensor coil. Therefore, erratic fluctuations in background noise interfere with the susceptibility signal in several intervals between 5 and 33 mbsf in Hole 762B and the interval 8-45 mbsf in Hole $763 \mathrm{~A}$. A negative susceptibility peak (about $-6 \times 10^{-6} \mathrm{cgs}$ ) was observed at 31 mbsf from Hole $762 \mathrm{~B}$, though there is no apparent change in lithology except that a significant amount (1\%) of zeolite (a nonmagnetic mineral) was identified in the smear slides in the interval between 23 and 33 mbsf where the clay content is very low. Biogenic carbonate and siliceous components are diamagnetic. They have a negative susceptibility value. Study of sediment magnetism (King et al., 1982) show that ferrimagnetic minerals (e.g., magnetite and titanomagnetite) dominate the magnetic properties of naturally occurring sediments. However, diamagnetic minerals could have a significant effect on the susceptibility measurements when ferrimagnetic mineral concentration is low. Silica is usually regarded as an indicator of biogenic material in a pelagic region (Dymond et al., 1977). This negative feature was not seen in susceptibility profile from Hole 763A, suggesting changes in biogenic processes.

\section{DISCUSSION}

In general, the paleomagnetic data from the Neogene sediments in Holes 762B and 763A appear reliable, especially from discrete samples with AF demagnetization types A, B, and $\mathrm{C}$. On the basis of nannofossil data (see "Biostratigraphy" section, "Site 762" and "Site 763" chapters, Haq, von Rad, O'Connell, et al., 1990), all reversals are correlated with the latest magnetic polarity time scale (Haq et al., 1988). The correlations based on magnetostratigraphy between the two holes are shown in Figure 5. The correlations based on the susceptibility measurements are also shown in Figures 6 and 7. To check the consistency of the correlations between the two different magnetic methods, a Shaw diagram of depth in Hole $763 \mathrm{~A}$ vs. depth in Hole $762 \mathrm{~B}$ was drawn (Fig. 8) by comparing the correlations shown in Figure 5 with those made in Figures 6 and 7. Figure 8 shows a very good consistency between the two methods.

The sequence of about 37 reversals in Holes 762B and 763A (Table 3) yields the age-depth curve shown in Figure 9A. Differentiation of these curves gives sedimentation rate as a function of time (Fig. 9B). The age-depth curves for Holes $762 \mathrm{~B}$ and $763 \mathrm{~A}$ show a similar trend except that the sedimentation rate in Hole $763 \mathrm{~A}$ is slightly higher than that in Hole 762B. In both holes, there has been decreased sedimentation in the last $3.5 \mathrm{~m} . y$., implying a similar depositional environment. The sedimentation rate at both holes has been increasing since $6.8 \mathrm{Ma}$. The highest sedimentation rate for Hole $762 \mathrm{~B}(\sim 20 \mathrm{~m} / \mathrm{m} . \mathrm{y}$.) occurred at $\sim 4.6 \mathrm{Ma}$ and for Hole $763 \mathrm{~A}$ $(\sim 24 \mathrm{~m} / \mathrm{m}$.y.) at $3.3 \mathrm{Ma}$. The periods of maximum sedimentation rate correlate with high values of susceptibility $(10-15 \times$ $10^{-6} \mathrm{cgs}$ ) in the intervals of $40-50$ mbsf in Hole 762B and $55-65$ mbsf in Hole $763 \mathrm{~A}$, which also indicates depositional control on the intensity of susceptibility.

I believe the changes in both NRM intensity and susceptibility from the Holes $762 \mathrm{~B}$ and $763 \mathrm{~A}$ reflect changes in concentration of magnetic materials supplied to the sediments. In Figure 6, the susceptibility, iron content, and aluminum content (from De Carlo, this volume) are plotted against depth for both holes. Figure 10 shows the relationship between susceptibility and iron content for Holes 762B and $763 \mathrm{~A}$. The reasonably good correlations shown in Figure 10 support the hypothesis that magnetic concentration observed at both holes reflects fluctuations in terrigenous influx. Based 


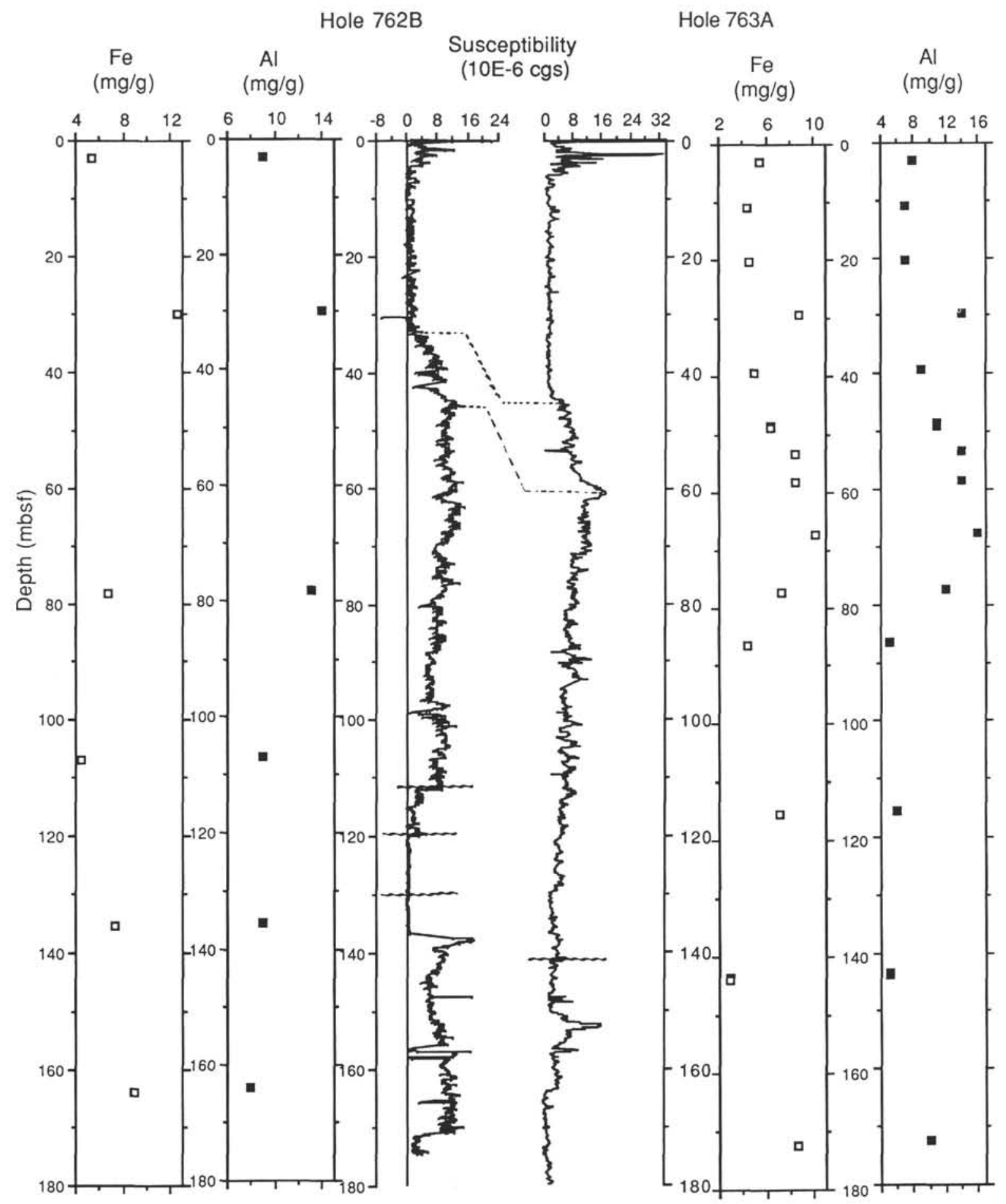

Figure 6. Whole-core susceptibility profile, iron content, and aluminum content vs. depth for Holes $762 \mathrm{~B}$ and $763 \mathrm{~A}$.

on the paleoceanographic record of the Maurice Ewing Bank (Ciesielski et al., 1982), one can speculate that climatic changes accompanied by a reduction in the vigor of oceanic circulation may cause an abrupt decrease in the supply of magnetic material from continental sources.

Evidence supporting the existence of an association between magnetic mineral concentration and climatic variation is strongest in the interval between 115 and 135 mbsf in Hole 762B (Fig. 6). The susceptibility in this interval is extremely low and three major hiatuses are present. Studies of oxygen and carbon isotopes are needed to support this speculation. Moreover, at $137-145$ mbsf in Hole 762B and at $150-165 \mathrm{mbsf}$ in Hole 763A, the susceptibility is fairly high $\left(\sim 5\right.$ to $15 \times 10^{-6}$ cgs). The interval of high magnetic susceptibility coincides with the well known early Miocene ( $21-24$ m.y.) lowstand period of eustatic level (Haq et al., 1988). Although inconclusive, this relationship supports the suggestion that the NRM intensity and magnetic susceptibility profiles observed in Holes 762B and 763A reflect changes in depositional processes associated with climatic change. 


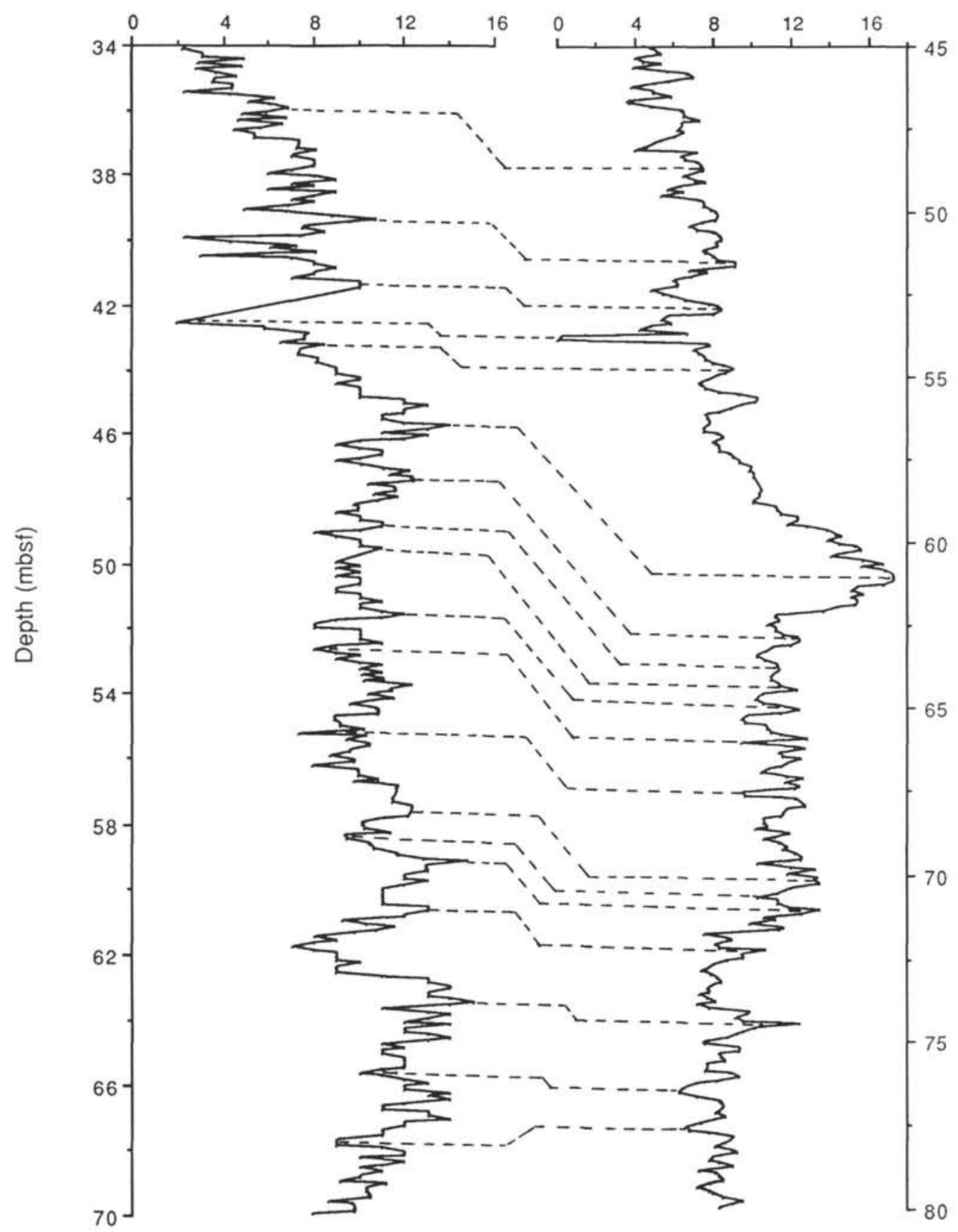

Figure 7. Suggested correlations between the whole-core magnetic susceptibility profiles of the middle portions of Hole 762B and 763A.

\section{CONCLUSIONS}

This study contains a record of magnetostratigraphy of the Neogene sediments from Holes 762B and 763A. Approximately 40 reversals are found and can be correlated to the magnetic polarity time scale, yielding an age-depth curve for each hole.

Hole $762 \mathrm{~B}$ and $763 \mathrm{~A}$ both appear to have had a similar sedimentation rate prior to $6.8 \mathrm{Ma}$. In Hole $762 \mathrm{~B}$, the sedimentation rate at $6.8 \mathrm{Ma}$ was $14 \mathrm{~m} / \mathrm{m}$.y. It then increased uniformly to a maximum value of about $20 \mathrm{~m} / \mathrm{m}$.y. at $4.6 \mathrm{Ma}$. Following a fairly uniform decay, it decreased to a minimum value of $13 \mathrm{~m} / \mathrm{m} . \mathrm{y}$. at $0.7 \mathrm{Ma}$. Similarly, the sedimentation rate in Hole $763 \mathrm{~A}$ reached a maximum value of $24 \mathrm{~m} / \mathrm{m}$.y. at 3.3 $\mathrm{Ma}$ and decreased to a minimum value of $18 \mathrm{~m} / \mathrm{m}$.y. with fairly large fluctuations.

The fluctuations of the susceptibility and NRM intensity observed at both holes correlate well with changes in lithology and sedimentation rate of sediments since $6.8 \mathrm{Ma}$. This correlation suggests changes in the supply of the terrigenous influx to the sites, probably controlled by depositional changes. In at least one instance, these changes seem to correlate with a climatic change. 


\section{ACKNOWLEDGMENTS}

I sincerely thank E. De Carlo for providing his unpublished geochemistry data, P. Haeussler and R. S. Coe for helpful discussions, S. O'Connell and the reviewers for their helpful reviews and many valuable suggestions, and the Joint Oceanographic Institutions, Inc., for partial financial support.

\section{REFERENCES}

Berggren, W. A., Kent, D. V., and Van Couvering, J. A., 1985. The Neogene: Part 2. Neogene geochronology and chronostratigraphy. In Snelling, N. J. (Ed.), The Chronology of the Geological Record. Geol. Soc. London Mem., 10:211-260.

Bloemendal, J., King, J. W., Tauxe, L., and Valet, J.-P., 1989. Rock-magnetic stratigraphy of Leg 108 Sites 658, 659, 661, and 665 , eastern tropical Atlantic. In Ruddiman, W., Sarnthein, M., et al., Proc. ODP, Sci. Results, 108: College Station, TX (Ocean Drilling Program), 415-428.

Bloemendal, J., Lamb, B., and King, J., 1988. Paleoenvironmental implications of rock-magnetic properties of late Quaternary sediment cores from the eastern equatorial Atlantic. Paleoceanography, 3:61-87.

Ciesielski, P. F., Ledbetter, M. T., and Ellwood, B. B., 1982. The development of Antarctic glaciation and the Neogene paleoenvironment of the Maurice Ewing Bank. Mar. Geol., 46:1-51.

Doh, S.-J., King, J., and Lienen, M., 1988. A rock-magnetic study of GPC-3 from the central North Pacific and its paleoceanographic implications. Paleoceanography, 3:89-112.

Dymond, J., Corliss, J. B., and Health, G. R., 1977. History of metalliferous sediments at DSDP site 319 in the south eastern Pacific. Geochim Cosmochim. Acta, 41:747.

Hall, F. R., Bloemendal, J., King, J. W., Arthur, M. A., and Aksu, A. E., 1989. Middle to Late Quaternary sediment fluxes in the Labrador Sea, ODP Leg 105, Site 646: a synthesis of rockmagnetic, oxygen-isotopic, carbonate, and planktonic foraminif- eral data. In Srivastava, S. P., Arthur, M., Clement, B., et al., Proc. ODP, Sci. Results, 105: College Station, TX (Ocean Drilling Program), 653-688.

Haq, B. U., Hardenbol, J., and Vail, P. R., 1988. Mesozoic and Cenozoic chronostratigraphy and cycles of sea-level change. In Wilgus, C., et al., (Eds.), Sea-Level Change-An Integrated Approach. Soc. Econ. Paleontol. Mineral. Spec. Publ., 42:71-108.

Haq, B. U., von Rad, U., O'Connell, S., et al., 1990. Proc. ODP, Init. Repts., 122: College Station, TX (Ocean Drilling Program).

Hays, J. D., Saito, T., Opdyke, N. D., and Burckle, L. H., 1969. Pliocene-Pleistocene sediments of the equatorial Pacific: their paleomagnetic, biostratigraphic, and climatic record. Geol. Soc. Am. Bull., 80:1481-1513.

Kennett, J. P., 1982. Marine Geology: Englewood Cliffs, NJ (Prentice Hall).

King, J. W., 1986. Paleomagnetic and rock-magnetic stratigraphy of Pigmy Basin, Deep Sea Drilling Project Site 619, Leg 96. In Bouma, A. H., Coleman, J. M, Meyer, A. W., et al., Init. Repts. DSDP, 96: Washington (U.S. Govt. Printing Office), 677-684.

King, J. W., Leskee, W., Marvin, J., and Banerjee, S. K., 1982. Identification of magnetite and hematite in natural samples: a comparison of different rock magnetic methods. Eos, 63:917.

Opdyke, N. D., Burckle, L., and Todd, A., 1974. The extension of the magnetic time scale in sediments of the Central Pacific Ocean. Earth Planet. Sci. Lett., 22:300-306.

Robison, S. G., 1986. The Late Pleistocene paleomagnetic record of North Atlantic deep-sea sediments revealed by mineral-magnetic measurements. Phys. Earth Planet. Inter., 42:22-47.

Thompson, R., Bloemendal, J., Dearing, J. A., Oldfield, F., Rummery, T. A., Stober, J. C., and Turner, G. M., 1980. Environmental applications of magnetic measurements. Science, 207:481-486.

Date of initial receipt: 4 May 1990

Date of acceptance: 10 December 1990

Ms 122B-153 


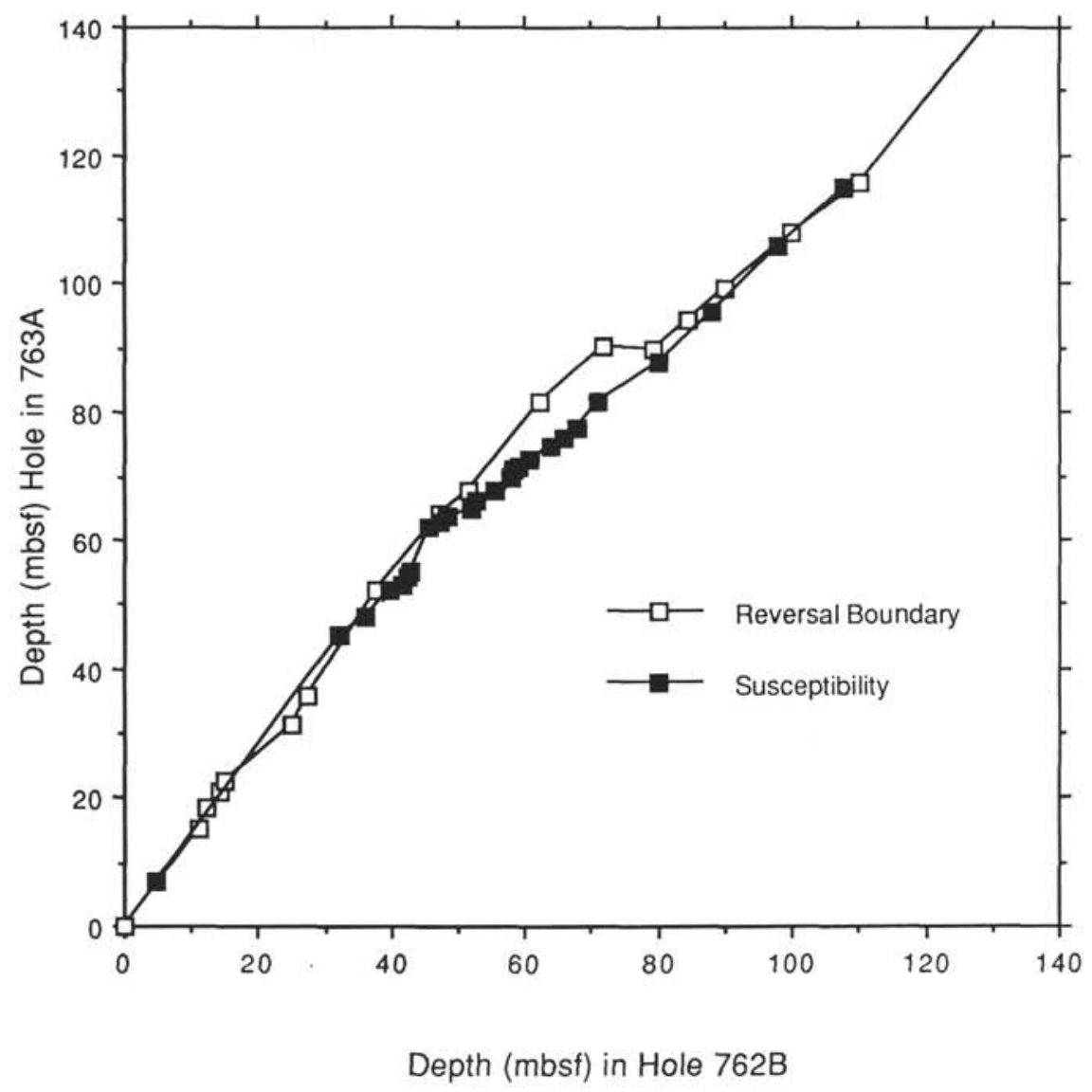

Figure 8. Shaw correlation diagram showing the relationship between the lithostratigraphic records of Holes $762 \mathrm{~B}$ and $763 \mathrm{~A}$, based on magnetostratigraphy and susceptibility of each hole. 

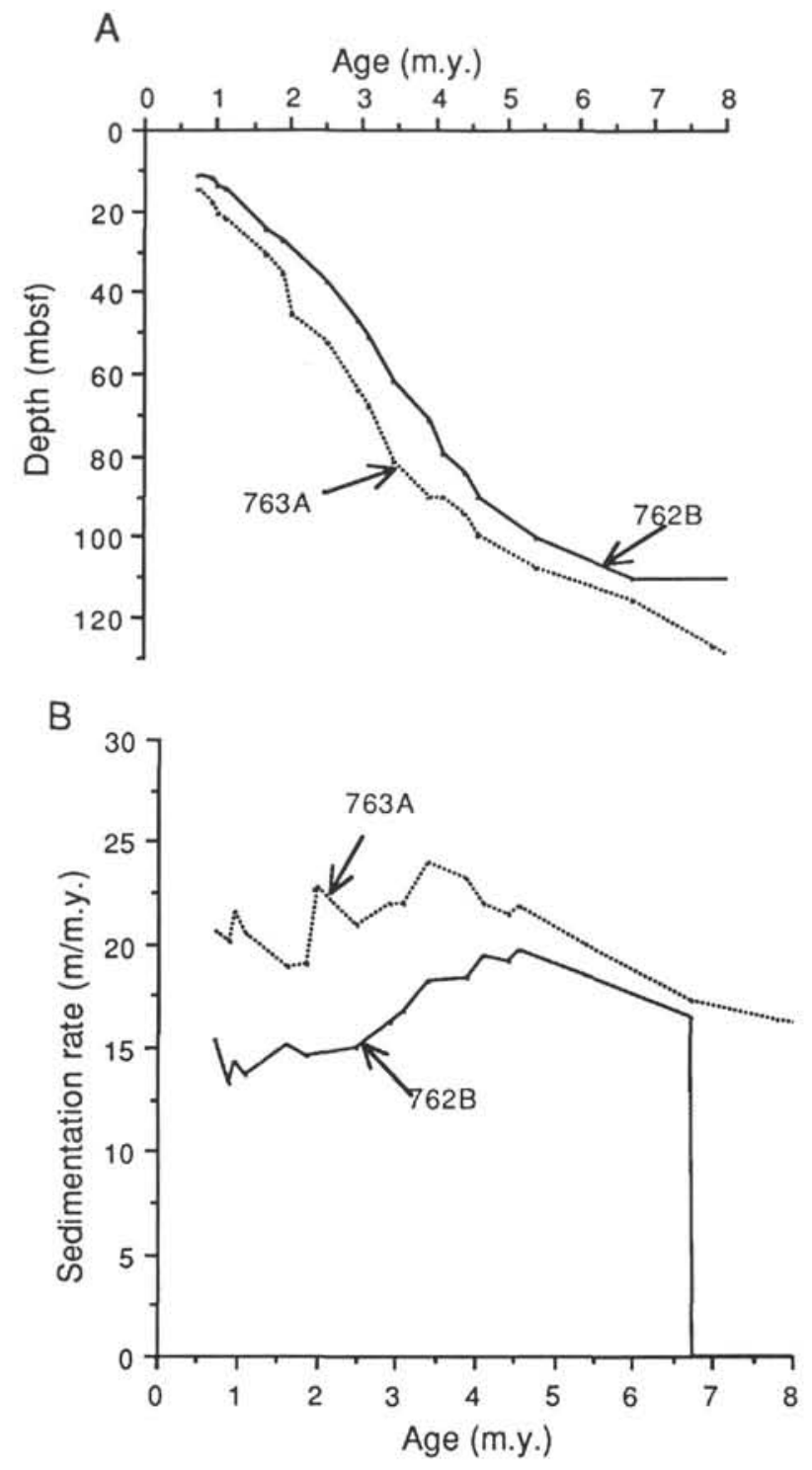

Figure 9. A. Age-depth curve. B. Sedimentation rate as a function of age for Holes 762B and 763A. 

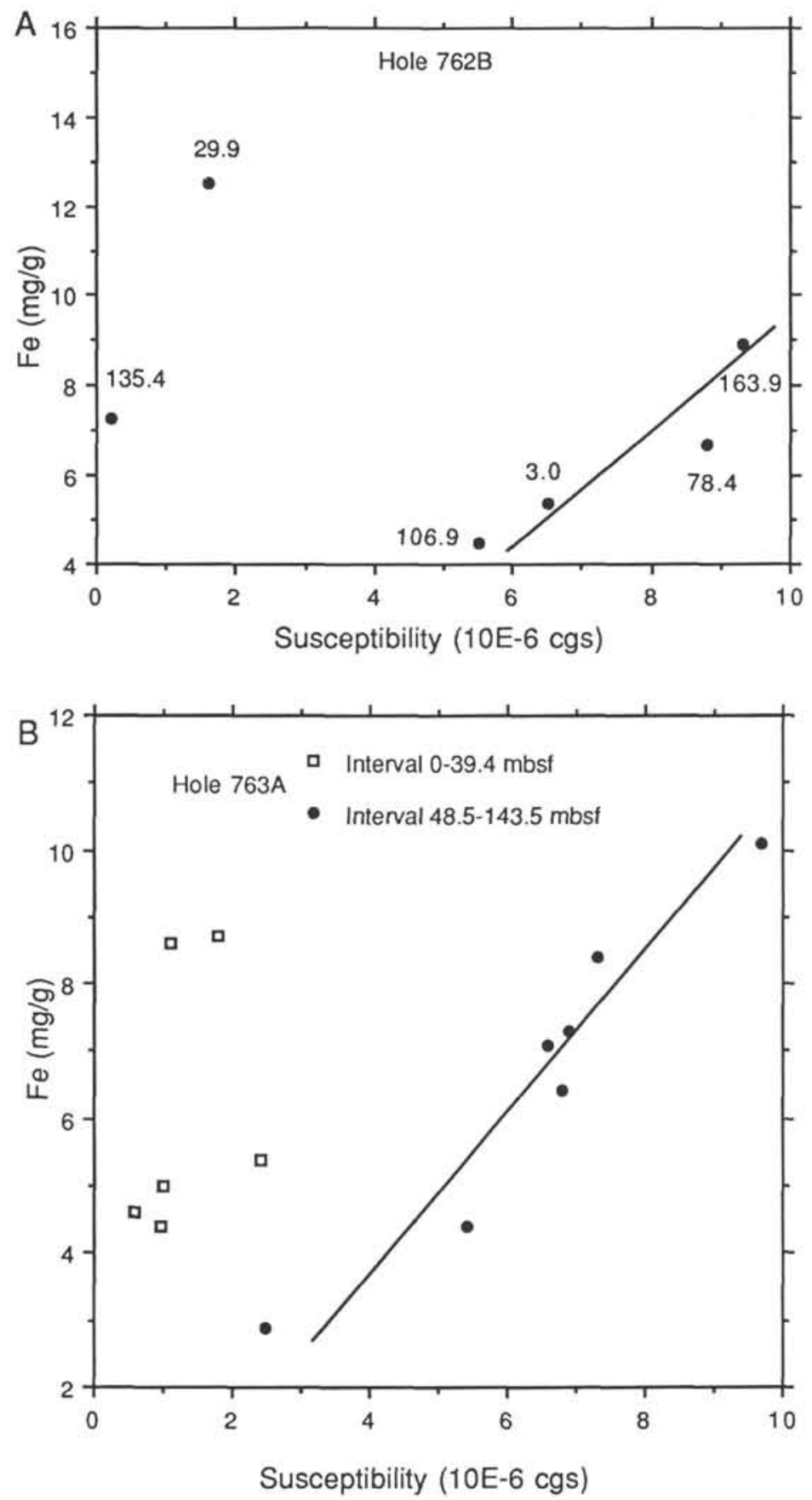

Figure 10. Whole-core susceptibility vs. iron content (De Carlo, this volume) for (A) Hole 762B and (B) Hole 763A, showing positive correlations between susceptibility and concentration of terrigenous material in the interval $48.5-143.5$ mbsf in Hole 763A. 